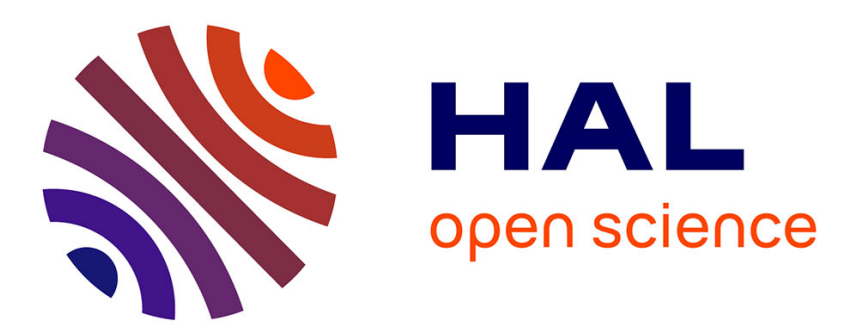

\title{
Relative astrometry and near-infrared differential photometry of nearby southern orbital binaries with adaptive optics
}

\author{
Patricia Lampens, Jean-Louis Prieur, R.W. Argyle, J. Cuypers
}

\section{To cite this version:}

Patricia Lampens, Jean-Louis Prieur, R.W. Argyle, J. Cuypers. Relative astrometry and near-infrared differential photometry of nearby southern orbital binaries with adaptive optics. Astronomical Notes / Astronomische Nachrichten, 2012, 334 (3), pp.237-250. 10.1002/asna.201211724 . hal-02903259

\section{HAL Id: hal-02903259 \\ https://hal.science/hal-02903259}

Submitted on $20 \mathrm{Jul} 2020$

HAL is a multi-disciplinary open access archive for the deposit and dissemination of scientific research documents, whether they are published or not. The documents may come from teaching and research institutions in France or abroad, or from public or private research centers.
L'archive ouverte pluridisciplinaire HAL, est destinée au dépôt et à la diffusion de documents scientifiques de niveau recherche, publiés ou non, émanant des établissements d'enseignement et de recherche français ou étrangers, des laboratoires publics ou privés. 


\title{
Relative astrometry and near-infrared differential photometry of nearby southern orbital binaries with adaptive optics ${ }^{\star}$
}

\author{
P. Lampens ${ }^{1}$, J.-L. Prieur ${ }^{23, \star \star}$, R.W. Argyle ${ }^{4}$, and J. Cuypers ${ }^{1}$ \\ 1 Koninklijke Sterrenwacht van België, Ringlaan 3, B-1180 Brussel, België. \\ 2 Université de Toulouse; UPS-OMP; IRAP; Toulouse, France \\ ${ }^{3}$ CNRS; IRAP; 14 avenue E. Belin, 31400 Toulouse, France \\ 4 Institute of Astronomy, Madingley Road, Cambridge, CB3 0HA, United Kingdom
}

Received Sept. 19th 2012; accepted

Key words Astrometry - Photometry — Stars: Binaries: close - Binaries: visual

We present relative astrometry and differential photometry measurements for a sample of nearby southern orbital binaries making use of the technique of Adaptive Optics. The observations were made in December 2000, with the ADONIS camera mounted at the 3.6m ESO telescope from La Silla Observatory, equipped with the broad-band near-infrared filters $\left(J_{-}, H\right.$-, $K$-passbands). Our sample contains stars which do not fit very well the empirical mean mass-luminosity relation (according to our previous study), but for which accurate parallaxes (determined by the Hipparcos satellite) and high-quality orbits were available thanks to many previous efforts. We derived accurate positions and $J, H, K$ magnitudes of the individual components of those binaries. The individual stellar components have near-infrared colour indices well grouped in those plots and are comparable to standard single stars.

The data reduction procedure used for deriving those results is described in detail. It is based on a leastsquares fit of Moffat-Lorentz profiles in direct imaging for well-resolved systems and on Fourier analysis for very close pairs.

\section{Introduction}

Binaries with significant orbital motions deserve to be observed and studied because they are essential in deriving stellar masses in a fundamental and direct way. New stellar mass determinations are still much needed to enable detailed comparisons with models of stellar structure and evolution. The required accuracy for this purpose is of the order of $1-2 \%$ at least on the individual stellar mass (Andersen, 1991). This requirement is much more difficult to meet for the visual binaries (VB's) which are on the lower part of the Main Sequence than for the eclipsing binaries (EB's) due to the much longer orbital periods and the need for high-angular resolution to resolve the closest pairs. This is why the empirical Mass-Luminosity Relation (noted hereafter as MLR) remains poorly determined for masses $M$ less than $1 M_{\odot}$ (Henry \& McCarthy 1993; Henry et al., 1999; Delfosse et al., 2000 - for $M \leq 0.6 M_{\odot}$; Henry 2004 - from end to end with a special emphasis for $\left.M \leq 0.2 M_{\odot}\right)$. However, the class of visual binaries (including the "speckle" or interferometric binaries) is important for various reasons:

- visual binaries have non-interacting components in general. They thus represent better the evolutionary scenario of single stars;

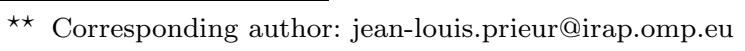

- they carry a lot of angular momentum and therefore represent a different fraction of the binary population than eclipsing binaries whose masses can be very accurately determined;

- they are the only objects which allow calibration of the MLR in the mass range from 0.2 to $1.0 M_{\odot}$;

- the distributions of their dynamical characteristics (orbital elements such as the period $P_{\text {orb }}$, the true semi major axis $A$ and eccentricity $e$ ) are directly connected to the mechanisms of their formation (Halbwachs et al., 2005, Patience et al., 1998).

Often, the limiting factor in the precise determination of the stellar mass is the poor knowledge of the distance of the system. Thanks to the astrometric satellite mission Hipparcos (ESA, 1997), many of the parallax uncertainties have been reduced by a factor of 2 to 5 . However, this improvement is generally not sufficient: after the Hipparcos mission, the principal error contribution on the mass determination of binary systems still comes from the error on the parallax. Indeed, according to Kepler's laws, the relative error on the total mass $M$ of the two components is given by $\varepsilon_{M}=\sqrt{\varepsilon_{a^{3} / P^{2}}^{2}+9 \varepsilon_{\pi}^{2}}$, where $a$ is the semi-major axis in AU, $P$ the period in yr, $M$ the sum of the masses in $M_{\odot}$, and $\pi$ the parallax in arcseconds, and where $\varepsilon$ designates a relative rms error (see for example, Lampens et al., 1997). 
In an early review on the determination of stellar masses, Popper (1980) listed only $8 V B$ 's with a relative error on the sum of masses less than $3 \%$. Twenty years later, thanks to the improvement of the parallax determination obtained by Hipparcos, Ruymaekers (1999) listed $30 \mathrm{VB}$ 's with a relative error on the ratio $a^{3} / P^{2}<3 \%$, while Söderhjelm (1999) reported only $10 V B$ 's with a relative error on the sum of masses smaller than $5 \%$ (cf. his tables 1 \& 2). This illustrates the difficulty for improving our knowledge on stellar masses in specific mass intervals (Xia et al. 2008 - for masses from $0.07 M_{\odot}$ to $\left.0.2 M_{\odot}\right)$.

But for a fully consistent picture of the system, the total mass determination is insufficient. Fractional masses as well as absolute luminosities are needed too. The relative error $\varepsilon_{\mathrm{M}}$ of the component absolute luminosity $\mathrm{M}$ again depends (largely) on the parallax accuracy $\varepsilon_{\pi}$, but also on the error of the component magnitude $\varepsilon_{m}$ according to $\varepsilon_{\mathrm{M}}=\sqrt{\left[\left(5 \cdot \log _{10} \mathrm{e}\right) \varepsilon_{\pi}\right]^{2}+\varepsilon_{m}^{2}}$ (where e is the base of the Napierian logarithm).

Accurate component magnitudes and colours are thus further needed to derive reliable physical properties of the components. The Hipparcos Catalogue provided for the first time a precise differential magnitude, $\Delta H_{p}$ for many visual binaries of sub-arcsecond separation (ESA, 1997; Martin \& Mignard, 1998), and the complementary Tycho Double Star Catalogue (Fabricius et al., 2002) also gave $\Delta B_{T}$ and $\Delta V_{T}$ for systems with $\rho>0^{\prime \prime} .5$ and $\Delta m<3$. Unfortunately, many nearby visual binaries were too close to be fully resolved by Hipparcos/Tycho and still lack precise component colours. Existing spectral classifications generally refer to the system as a whole: when component spectral types are reported, these usually rely on a visual estimation of the magnitude difference, with a typical error of the order of $\pm 0.2 \mathrm{mag}$ (Christy \& Walker, 1969; Edwards, 1976). Therefore, the situation is such that the (rare) precise stellar masses of visual binary systems are generally attributed to components with unknown or uncertain colours.

This point is well-illustrated in the study by Delfosse et al. (2000) on M-dwarf binaries with masses accurate to within $10 \%$. Their table 2 in which they collected basic information for 18 pairs, i.e. 3 EBs and 15 VBs, is lacking accurate magnitude differences in several cases: the flux ratios in the near-IR bands were not determined for 7 systems (3 EBs $+4 \mathrm{VBs})$, while 6 systems had spectroscopically derived magnitude differences, inferred from the relative line depths of doublelined spectra (1 EBs $+5 \mathrm{VBs})$, and 6 had large errors (0.1 mag or larger) on the visual magnitude difference $\Delta m_{V}(2 \mathrm{EBs}+4 \mathrm{VBs})$.

The aim of the present study is to determine differential near-infrared magnitudes and colours for a sample of nearby close visual binaries from a single observation with the technique of adaptive optics (AO).
This technique offers high-angular resolution and allows to derive reliable differential photometry between the components (ten Brummelaar et al. 1996; Roberts \& ten Brummelaar 1998; Roberts et al. 2005). In addition, it provides relative astrometric data useful for the computation of improved orbits.

\section{Description of the sample}

The following criteria were used for selecting the target binaries:

- nearby visual binaries (i.e., located within a distance $\leq 67 \mathrm{pc}$ ) for which the combination of accurate Hipparcos parallaxes (for which the relative error is well below 5\%) with high-quality orbits allows to derive component masses with an accuracy, $\varepsilon_{M}$ better than $10-15 \%$. This latter information is based on the catalogue of orbital parameters with realistic errors computed by means of the Bootstrap technique (Ruymaekers, 1999);

- with predicted angular separations between $0^{\prime \prime} .1$ and $1^{\prime \prime}$ at epoch 2001.0 and with declination less than $+10^{\circ}$;

- having orbital periods from 2 to $700 \mathrm{yr}$ and total masses from 0.4 to $8 M \odot$;

- which do not fit well the mean empirical MLR derived from the new Hipparcos parallaxes (Lampens et al., 1997)

To this list we also added some 18 binaries from table 1 of Söderhjelm (1999), with orbital periods less than $29 \mathrm{yr}$ and with new astrometric mass ratios but which were not always consistent with the previous determination (Martin \& Mignard, 1998).

This selection led to a list of some 50 bright, close southern visual binaries. The sub-sample of the objects from this list that we could observe is presented in Table 1 and described in the next section.

\section{Observations}

The observations were performed with the ESO 3.6-m telescope of La Silla, during two nights (13-14 and 1415 December 2000). The telescope was equipped with ADONIS ('ADaptive Optics Near Infrared System') and the Sharp II+ infrared camera.

We employed a lens scale of $\sim 0^{\prime \prime} .050 /$ pixel (see Sect. 4) and the ESO broad-band filters $J\left(\lambda_{c}=1.253\right.$ $\mu \mathrm{m}, \Delta \lambda=0.296 \mu \mathrm{m}), H\left(\lambda_{c}=1.643 \mu \mathrm{m}, \Delta \lambda=0.353 \mu \mathrm{m}\right)$ and $K\left(\lambda_{c}=2.177 \mu \mathrm{m}, \Delta \lambda=0.378 \mu \mathrm{m}\right)$. Exposure times ranged from $40 \mathrm{~ms}$ (shutter mode) to $500 \mathrm{~ms}$ (normal mode). We used the standard configuration with chopping mode between star and sky in order to optimize the number of exposures per star. In this way, for each filter, the observational data consisted of four sets of 20 exposures each: $2 \times 20$ exposures with the target 
Table 1 Basic information for the sample of observed systems

\begin{tabular}{|c|c|c|c|c|c|c|c|}
\hline HIP & $\begin{array}{l}\text { CCDM } \\
(\alpha, \delta)_{2000}\end{array}$ & $\begin{array}{c}\mathrm{Hp} \\
(\mathrm{mag})\end{array}$ & $\mathrm{SpT}$ & $\begin{array}{l}\pi_{H i p} \\
(\operatorname{mas})\end{array}$ & $\begin{array}{l}\mathrm{P}_{\text {orb }} \\
\text { (year) }\end{array}$ & $\begin{array}{l}\text { Discovery } \\
\text { Designation }\end{array}$ & Notes \\
\hline 730 & $00090-5400$ & 6.462 & G4IV.. & 7.00 & 221. & HDO 181 & GJ 9 \\
\hline 2237 & $00284-2020$ & 6.558 & G3V & 31.01 & $5.7 / 11.3$ & В 1909 & ambig. solution (ESA 1997) \\
\hline 2762 & $00352-0336$ & 5.321 & F6V & 47.51 & 6.9 & HO 212 & Gl 23, ADS 490 \\
\hline 2941 & $00373-2446$ & 5.713 & $\mathrm{~K} 1 \mathrm{~V}+\ldots$ & 64.38 & 25.1 & BU 395 & Gl 25; SB-VB \\
\hline 5165 & $01061-4643$ & 3.465 & G8III & 16.46 & 168. & SLR 1 & $\mathrm{DMSA} / \mathrm{X}$ \\
\hline 5842 & 01157-6852CD & 4.964 & $\mathrm{~K} 2 \mathrm{~V}$ & 48.94 & 85.2 & I 27 & Gl 55.1; MLR outlier \\
\hline 7254 & $01334-4354$ & 7.975 & $\mathrm{~K} 0 / \mathrm{K} 1 \mathrm{~V}$ & 28.23 & & HDS 205 & \\
\hline 7372 & $01350-2954$ & 7.253 & $\mathrm{~K} 3 \mathrm{~V}$ & 42.29 & 4.6 & DAW 31 & Gl 60; triple \\
\hline 7580 & $01376-0924$ & 6.355 & F7V & 26.15 & 29. & KUI 7 & DMSA/G; SB-VB \\
\hline 15382 & $03184-2231$ & 5.024 & K0III & 12.79 & 112.4 & SEE 23 & \\
\hline 19719 & $04136+0743$ & 5.374 & F3V ... & 27.04 & 7.2 & A 1938 & \\
\hline 20087 & $04184+2135 \mathrm{Aa}, \mathrm{Ab}$ & 5.713 & F0V & 18.25 & 11.3 & MCA $14 \mathrm{Aa}, \mathrm{Ab}$ & $51 \mathrm{Tau}, \mathrm{SB}$ \\
\hline 21280 & $04340+1510^{*}$ & 8.637 & G5 & 24.02 & 13. & CHR 17 & multiple? \\
\hline 22550 & $04512+1104$ & 6.866 & F6V & 20.15 & 16.6 & BU 883 & ADS 3475 , MLR outlier \\
\hline 22573 & $04515-3454$ & 5.863 & $\mathrm{~A} 2 / 3 \mathrm{~V}$ & 11.39 & 37.3 & FIN 320 & \\
\hline 22607 & $04518+1339$ & 6.365 & F5 & 23.91 & 95.1 & BU 552 & ADS 3483, SB, MLR outlier \\
\hline 25037 & $05217+1240$ & 8.032 & A3 & 7.39 & & STT 105 & ADS 3936 \\
\hline 25119 & $05226+0236$ & 7.903 & $\mathrm{~K} 3 \mathrm{~V}$ & 50.24 & 88.1 & A 2641 & ADS 3959 \\
\hline 27421 & $05484+2052$ & 6.020 & B9V & 5.48 & & STT 118 & ADS 4392 \\
\hline 27901 & $05542-2909$ & 6.430 & F3V & 17.84 & & FIN 382 & \\
\hline 29234 & $06098-2246$ & 5.808 & F5V & 18.48 & 18.3 & RST 3442 & MLR outlier \\
\hline 30920 & $06294-0249$ & 10.984 & $\mathrm{M} 4.5 \mathrm{Ve}$ & 242.89 & 16.5 & B 2601 & Flare star, DMSA/G, Gl 234 \\
\hline 30953 & $06298-5014$ & 5.308 & $\mathrm{~F} 2 \mathrm{~V}$ & 19.33 & 98.8 & R 65 & MLR outlier \\
\hline 33451 & $06573-3530$ & 6.333 & F5V & 23.15 & 16.8 & I 65 & Gl 255 \\
\hline 36238 & $07277+2127$ & 5.337 & F5IV-V ... & 29.38 & 2.0 & MCA 30Aa,Ab & 63 Gem, ADS 6089, MLR outlier \\
\hline 40167 & $08123+1738$ & 4.781 & G0V & 39.11 & 59.6 & STF 1196 & ADS $6650, \mathrm{SB}$ \\
\hline 41261 & $08251-4910$ & 7.801 & K0 & 30.14 & 25.6 & RST 321 & MLR outlier \\
\hline 42075 & $08345-3236$ & 6.550 & G5V & 15.93 & 17.3 & FIN 335 & giant? \\
\hline 45170 & $09123+1459 \mathrm{Aa}, \mathrm{Ab}$ & 6.626 & G9V & 48.83 & 2.7 & FIN 347 & $81 \mathrm{Cnc}, \mathrm{SB}-\mathrm{VB}$ \\
\hline 46404 & 09278-0604 & 5.504 & G2V & 32.01 & 33.8 & B 2530 & SB, giant? \\
\hline 46454 & $09285+0903$ & 5.532 & F9V & 29.05 & 117.0 & STF 1356 & $\omega$ Leo, SB, giant? \\
\hline 47479 & $09407-5759$ & 5.367 & A3IV & 14.85 & 10.6 & B 780 & GJ 9305; multiple? \\
\hline 53840 & $11009-4030$ & 6.886 & F7V & 15.11 & 4.5 & FIN 365 & \\
\hline 114375 & $23099-2227$ & 4.835 & $\mathrm{G} 2 \mathrm{IV}+\mathrm{A} 2$ & 6.26 & & RST 3320 & \\
\hline 114576 & $23126+0241$ & 7.839 & G5 & 15.21 & 29.2 & A 2298 & ADS 16591, SB-VB \\
\hline 115126 & $23191-1328 \mathrm{Aa}, \mathrm{Ab}$ & 5.245 & G6/G8IV & 48.22 & 6.3 & MCA 74Aa,Ab & $\begin{array}{l}94 \text { Aqr, ADS } 16672, \text { DMSA/X, } \\
\text { Gl 894.2, multiple? }\end{array}$ \\
\hline
\end{tabular}

* This flag in col. 2 indicates a WDS identifier (as no CCDM identifier was found)

binary on quadrant 1 , and $2 \times 20$ exposures with the target on quadrant 3 . The sky level was estimated on the quadrants 2 and 4, opposite to the quadrants used for the target. In nearly half of the cases, a single reference star was observed immediately after the binary to calibrate its point-spread-function (PSF).

Some 36 close visual binaries, most of them belonging to the all-sky sample described in Sect. 2, were observed during the allocated nights. They are listed in Table 1 with basic information including various identifiers (Cols. 1, 2, and 6), Hipparcos magnitude
Hp (Col. 3), spectral type (Col. 4), Hipparcos parallax (Col. 5), orbital period (Col. 6), (in years) and additional notes in Col. 8 (e.g. SB-VB: spectroscopic-visual binary). Unless specified in Col. 2, they all refer to AB couples. Among those, 11 targets were taken from table 1 from Söderhjelm (1999) and 9 are outliers from the empirical MLR with $\varepsilon_{M}<15 \%$. We also added a few targets needed for the astrometric calibration.

Because some of our main targets were not accessible during these nights, we completed the observations by inserting additional problematic targets 
though they had $\varepsilon_{M}>15 \%$. For example, we included HIP 7254 which is a new Hipparcos discovery and HIP 2237 for which "two orbital solutions fit the available data equally well: in the case of the higheccentricity, half-period solution a too high mass-sum is derived" (Söderhjelm, 1999). Unluckily, the latter remained unresolved.

On average the seeing was about $1-2^{\prime \prime}$, but the atmospheric conditions were more stable during the first night. This explains why ADONIS performed better and faster, resulting into a higher detection rate on the first night than on the second night. We were able to resolve 17 objects of the 20 binary systems observed on Dec. 13th 2000 whereas only 11 objects of the 17 observed on Dec. 14th 2000 (see Tables 2 and 3 in Sect. 5).

\section{Data reduction}

The reduction steps were performed in the following order:

1 - Preprocessing: bad pixel correction (darks), skysubtraction, flat-field division (using sky flats taken at twilight) were done using the excellent ESO Eclipse software package (Devillard, 1997).

2 - a) When the components were clearly resolved, we used a profile-fitting algorithm on each single frame. It is based on the fitting of twodimensional Moffat-Lorentz profiles (see Fig. 1 and Sect. 4.1).

b) When the binaries were not well-resolved, we performed a Fourier analysis of the power spectrum of the AO images. We calibrated the transfer function of the imaging system with observations of (unresolved) PSF reference stars located within $\sim 5^{\circ}$ from the binary targets. The ratio of the corresponding power spectra exhibited fringes when two stars were detected (e.g. Fig. 2). The determination of the position angle $\left(\theta_{0}\right)$ and period $\left(d_{0}\right)$ of those fringes then led to the astrometric parameters of the binary: $\rho=n / d_{0}$ (with $n \times n$ the size of the images), and $\theta=\theta_{0}+\pi / 2$. In this case, the position angle $\theta$ was determined with a $180^{\circ}$ ambiguity, which could sometimes be solved by direct inspection of the AO images.

3 - We used the astrometric calibration from Berthier et al. (2002) based on observations of the Trapezium cluster in the nights preceding and following our observations (since too few of our calibration double stars fitted the available field). We thus adopted the scale of $0^{\prime \prime} .0496 /$ pixel and the orientation of $269^{\circ} .609$ and $179^{\circ} .619$ for the $\mathrm{X}$ and $\mathrm{Y}$ axes, respectively (In Figs. 1 and 2, North is to the bottom, East to the left).

\subsection{Fitting procedure of Moffat profiles to long exposures}

Experience has shown that the point spread function of long exposure images is generally well represented by the semi-empirical Moffat-Lorentz profile (Moffat, 1982):

$$
I_{1}(x, y)=\frac{h_{1}}{\left(1+b r_{1}^{2}\right)^{q}}
$$

where $(x, y)$ are the Cartesian coordinates of the image, $q$ is a real value, generally in the range $[1,2]$ (it is a Lorentz profile when $q=2$ ), and $r_{1}$ is the distance of the current point $(x, y)$ to the centre of the profile $\left(x_{1}, y_{1}\right)$. The parameter $b$ is used to take into account the profile width. It is related to $s$, the full width at half maximum of the profile (FWHM or "seeing") with:

$$
s=\frac{2 \sqrt{2^{1 / q}-1}}{b}
$$

This profile was originally proposed by Moffat to model stellar profiles of photographic plates, to take into account the diffusion of light inside photographic emulsions. It was then widely used for many other applications and appeared to be well suited to images obtained with electronic detectors. In particular, we used this model for extracting the relative positions and differential magnitudes of CCD images of binary stars when profiles of the components overlap (Oblak et al., 1997, Lampens \& Strigachev, 2001, Lampens et al., 2001, Strigachev \& Lampens, 2004, and Lampens et al., 2007).

To obtain the differential photometry and relative astrometry measurements presented in Table 2, we have fitted the following brightness function corresponding to the sum of two Moffat two-dimensional profiles, to the ADONIS images:

$$
I(x, y)=\sum_{m=1}^{2} I_{m}(x, y)+n(x, y)
$$

with

$$
I_{m}(x, y)=\frac{h_{m}}{\left[1+\left(b r_{m}\right)^{2}\right]^{q}}
$$

where $m=1,2$ are the indices of the two stellar components. For each profile, $h_{m}$ is the central intensity and $r_{m}$ is the distance of the current point to the centre $\left(x_{m}, y_{m}\right)$ of the profile. The function $n(x, y)$ is a two dimensional polynomial that is used to model the sky background. For our application, a first order polynomial was sufficient:

$$
n(x, y)=n_{0}+n_{1} x+n_{2} y+n_{3} x y
$$

For the images where the profile isophotes of many frames were not circular, we took their ellipticity into account by changing the expression of $r_{m}$ :

$r_{m}=\sqrt{\left(x-x_{m}\right)^{2}+e\left(x-x_{m}\right)\left(y-y_{m}\right)+(1+k)\left(y-y_{m}\right)^{2}}$ 
where $e$ and $k$ are two additional parameters.

The problem to be solved consists therefore in minimizing a function $f(\boldsymbol{p})$ where the vector $\boldsymbol{p}$ belongs to $\mathbb{R}^{14}$. The corresponding 14 coordinates are $\left(x_{m}, y_{m}, h_{m}\right)$ (for $\left.\mathrm{m}=1,2\right), b, q, k, e, n_{0}, n_{1}, n_{2}$ and $n_{3}:$

$$
f(\boldsymbol{p})=\sum_{(x, y) \in \mathcal{A}}\left[z(x, y)-I_{\boldsymbol{p}}(x, y)\right]^{2}
$$

In this expression, $z(x, y)$ is the value of the brightness function as measured on the detector and $\mathcal{A}$ is the image domain within which the minimisation is performed.

This method was first implemented by one of us (JC) as a procedure in the ESO/MIDAS software, using a Newton-Raphson minimisation algorithm (J. Cuypers, 1997). For this application, we also developed a new stand-alone program fit_moffat.cpp, with the bound constrained LBFGS minimisation algorithm (Byrd et al, 1995). Both programs require the knowledge of the gradient vector $g$ of $f(\boldsymbol{p})$, whose components $g_{i}$ are given by:

$$
g_{i}=\sum_{(x, y) \in \mathcal{A}}-2\left[z(x, y)-I_{p}(x, y)\right] \frac{\partial I_{p}(x, y)}{\partial p_{i}}
$$

In appendix, we provide the expression of the gradient $\frac{\partial I_{p}(x, y)}{\partial p_{i}}$ that may be useful for the readers who want to create their own fitting program.

\section{Main results}

The relative astrometric and differential near-infrared photometric measurements obtained with the method of Moffat profile fitting for 32 components of 29 binary and multiple systems are listed in Table 2. For each couple we indicate its HIP number in Col. 1, and the component names in Col. 2, the Besselian epoch of observation in Col. 3 (in Besselian years, after subtraction from 2000), the number $N$ of quadrants used for the astrometric measurements in Col. 4 , the average relative astrometric measurements $\rho_{\text {all }}$ (Col. 5), $\theta_{\text {all }}$ (Col. 7), and their corresponding errors $\sigma_{\text {oall }}$ (Col. 6) and $\sigma_{\theta a l l}$ (Col. 8). The subscript 'all' is added to indicate that the current measurement corresponds to the average of all the measurements obtained with the three filters $J, H$, and $K$. In the next columns we report the differential photometric measurements $\Delta m_{J}, \Delta m_{H}$, and $\Delta m_{K}$ with their corresponding errors $\sigma_{\Delta m_{J}}, \sigma_{\Delta m_{H}}$, and $\sigma_{\Delta m_{K}}$, and the numbers of quadrants used for those photometric measurements: $N_{J}, N_{H}$, and $N_{K}$, respectively.

Table 3 lists the values we obtained using the method of Fourier analysis, i.e., for 8 resolved components of 6 multiple systems. For each system we indicate its HIP number in Col. 1, the component names in Col. 2, the fractional Besselian epoch of observation and the relative astrometric measurements $\rho, \theta$ and their corresponding errors $\sigma_{\rho}, \sigma_{\theta}$ for each filter $J$, $H$, and $K$. The subscript 'all' indicates that the current measurement corresponds to the average of the measurements in the different filters.

\subsection{Analysis of astrometric data}

The distribution of the angular separations $\rho_{\text {all }}$ reported in Tables 2 and 3 is plotted in Fig. 3. The average rms errors for $\left(\rho_{\text {all }}, \theta_{\text {all }}\right)$ are $\left(0^{\prime \prime} .008,0^{\circ} .8\right)$ and $\left(0^{\prime \prime} .010,2^{\circ} .3\right)$ for Moffat profile fitting and Fourier analysis, respectively. In the case of Fourier analysis, the larger error on $\theta_{\text {all }}$ is due to the difficulty of determining the direction of the fringes accurately (see Sect. 4).

We detected a new candidate binary star among our (supposed single) reference stars, HIP 19233, which was resolved in all the direct images obtained with the $J H$ and $K$ filters (see Table 2). This object (also known as HR 9233) is also the high proper-motion nearby star LTT 1877 (parallax 42.3 mas). This new pair has a magnitude difference close to $3 \mathrm{mag}$ (in $J H$ and $K$ ), which may explain why it was not previously resolved. Further observations are needed to confirm its nature.

The technique of Fourier analysis (see Sect. 4) allowed the measurement of angular separations close to the diffraction limit of the $3.6 \mathrm{~m}$ telescope (which is $0 . " 069$ in $J$, i.e., $\lambda / D$ ). In particular, this analysis revealed the presence of possible components $\mathrm{Ab}, \mathrm{Ac}$ and Ad around HIP 20087Aa that could not be measured with the Moffat profile fitting procedure. Components Ac and Ad would be newly-discovered components, located very close to the primary star, nearly at the theoretical diffraction limit (see Fig 2). Further confirmation is needed, especially for the Ad component which was only detected with the $J$ filter. The latter could be an artifact caused by a bad estimation of the PSF (let us recall that the PSF could only be calibrated by observations of single stars before or after the observation of our targets, see Sect. 4). The existence of the Ac component is more secure since it was detected at the same location, both in $H$ and $K$.

Table 4 illustrates the comparison of our relative positions with the positions computed at the given Besselian epoch (B 2000.95) for those systems which have a published orbit. For each measurement taken from Tables 2 and 3, we report the HIP number in Col. 1, the component names in Col. 2, the astrometric measurements $\rho_{\text {all }}, \theta_{\text {all }}$ in Cols. 4 and 6 , with their corresponding errors $\sigma_{\text {pall }}, \sigma_{\theta \text { all }}$ in Cols. 5 and 7 , and the numbers of quadrants used for those measurements in Col. 3. We write the HD number in italic in Col. 1 when the measurement was taken from Table 3, else it was taken from Table 2. In Cols. 8 and 9, we report the computed positions $\rho_{c}, \theta_{c}$ at the epoch of observation, from the orbit whose bibliographic reference is indicated in 

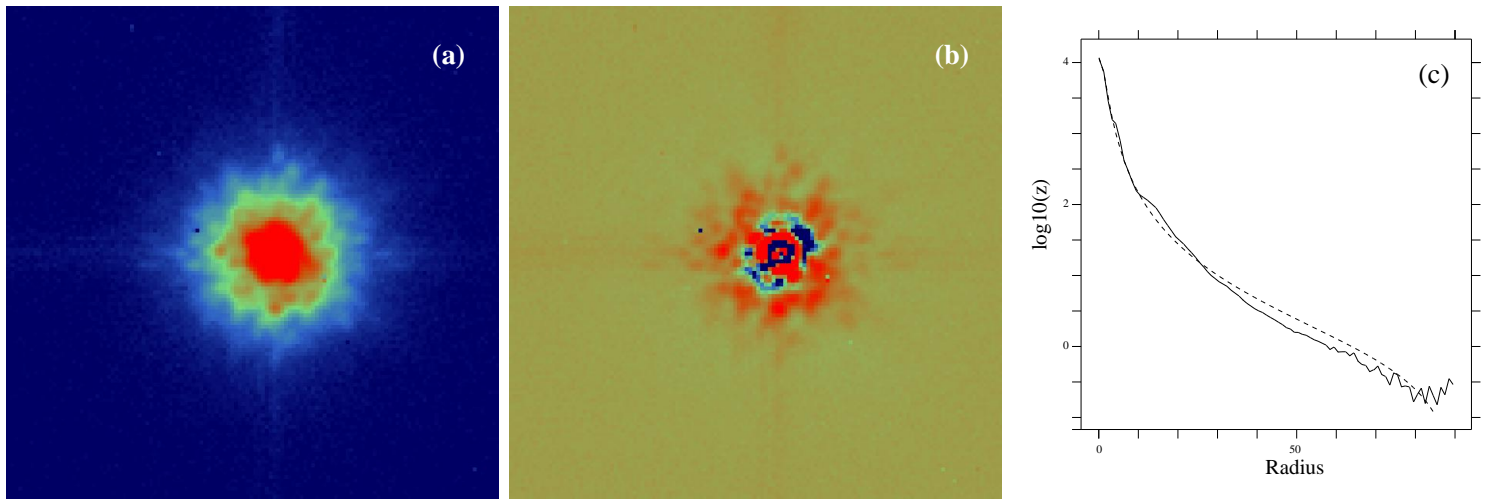

Fig. 1 Example of a Moffat-Lorentz profile fitted to the profile of the ADONIS image of calibration star HR 8658 obtained in $K$ : raw image (a), residual image (b) and comparison of the corresponding profiles (c), with the raw image profile (solid line) and the Moffat-Lorentz profile (dashed line).
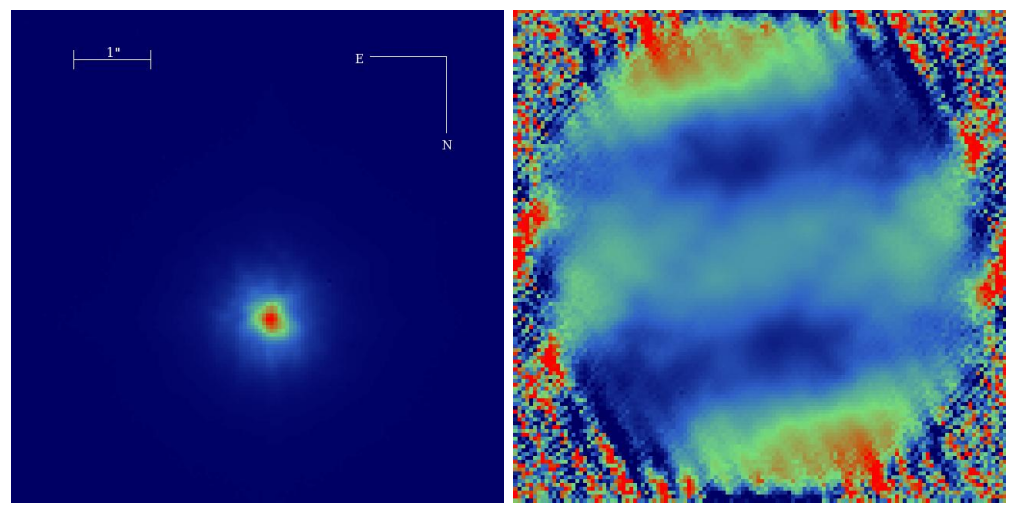

Fig. 2 Processing example of the image of HIP 20087 in $H$. The AO correction leads to integrated frames (left panel) which only show a single bright patch. When dividing the corresponding power spectrum with that of a calibration star, we obtain a "restored" power spectrum (right panel) which exhibits two systems of fringes betraying the presence of two companions 'Ab' and 'Ac' around the primary star (named 'Aa'). The inner disk that clearly appears in this power spectrum is bounded by the circle corresponding to the maximum resolution that can be reached with the 3.6-m telescope in $H$. The structure of the noise which is present outside of this disk also perturbs the "useful" signal inside of this disk.

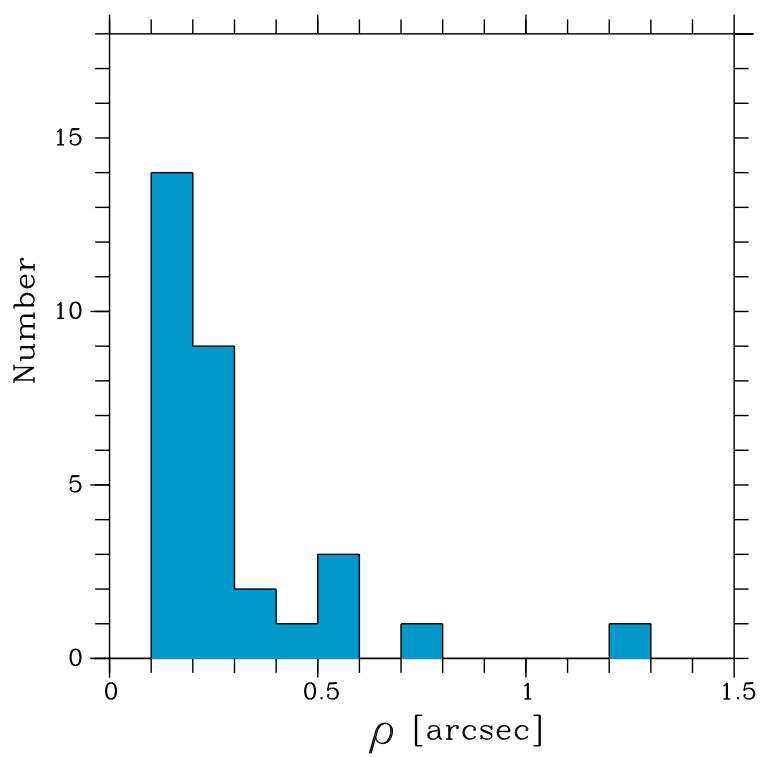

Fig. 3 Distribution of the angular separations reported in Tables 2 and 3
Col. 14. The corresponding residuals for $\rho, \theta$ are given in Col. 10 and 11, respectively. The position difference in Cartesian coordinates $\Delta$ Pos is indicated in arcseconds in Col. 12 , and the percentual ratio $\Delta \mathrm{Pos} / \rho_{\text {all }}$ is displayed in Col. 13. Most of the orbits used here were taken from the "Sixth Catalog of Orbits of Visual Binary Stars" (Hartkopf et al, 2012b), but we also added some orbits computed by Ruymaekers (1999) and a few more found from in the recent literature.

The residuals in the form $\rho . \Delta \theta_{O-C}$ versus $\Delta \rho_{O-C} / \rho$ are plotted in Fig. 4. The largest residuals are noticeable for HIP $7372 \mathrm{AC}$ and HIP 46404, indicating that their orbits are only preliminary. When using the criterion $\Delta \operatorname{Pos} / \rho_{\text {all }}>13 \%$ (see Col. 13 of Table 4 , two more objects appear with large residuals: HIP 20087 and HIP 27901, indicating that a revision of the orbit will also be needed in the future. In all other cases, the residuals are smaller than $13 \%$, which is an indication that our measurements agree rather well with the adopted ephemeris. 
Table 2 Relative astrometry and differential photometry obtained with Moffat profile fitting.

\begin{tabular}{|c|c|c|c|c|c|c|c|c|c|c|c|c|c|c|c|c|}
\hline HIP & $\mathrm{Cp}$ & $\begin{array}{l}\text { Epoch } \\
(2000 .)\end{array}$ & $\mathrm{N}$ & $\begin{array}{l}\rho_{\text {all }} \\
\left({ }^{\prime \prime}\right)\end{array}$ & $\begin{array}{c}\sigma_{\rho a l l} \\
\left({ }^{\prime \prime}\right)\end{array}$ & $\begin{array}{c}\theta_{\text {all }} \\
\left({ }^{\circ}\right)\end{array}$ & $\begin{array}{c}\sigma_{\theta a l l} \\
\left({ }^{\circ}\right)\end{array}$ & $\mathrm{N}_{J}$ & $\begin{array}{l}\Delta m_{J} \\
(\mathrm{mag})\end{array}$ & $\begin{array}{l}\sigma_{\Delta m_{J}} \\
(\mathrm{mag})\end{array}$ & $\mathrm{N}_{H}$ & $\begin{array}{l}\Delta m_{H} \\
(\mathrm{mag})\end{array}$ & $\begin{array}{l}\sigma_{\Delta m_{H}} \\
(\mathrm{mag})\end{array}$ & $\mathrm{N}_{K}$ & $\begin{array}{l}\Delta m_{K} \\
(\mathrm{mag})\end{array}$ & $\begin{array}{l}\sigma_{\Delta m_{K}} \\
(\mathrm{mag})\end{array}$ \\
\hline 730 & $\mathrm{AB}$ & .953 & 11 & 0.281 & 0.022 & 59.6 & 5.0 & 4 & 2.39 & 0.03 & 4 & 2.75 & 0.10 & 3 & 2.92 & 0.11 \\
\hline 2941 & $\mathrm{AB}$ & .956 & 12 & 0.554 & 0.003 & 292.5 & 0.4 & 4 & 0.26 & 0.02 & 4 & 0.24 & 0.02 & 4 & 0.25 & 0.04 \\
\hline 5165 & $\mathrm{AB}$ & .956 & 4 & 0.241 & 0.002 & 238.3 & 0.6 & - & - & - & - & - & - & 4 & 0.32 & 0.04 \\
\hline 5842 & CD & .956 & 4 & 0.947 & 0.011 & 275.8 & 0.2 & - & - & - & - & - & - & 4 & 0.29 & 0.02 \\
\hline 7254 & $\mathrm{AB}$ & .953 & 12 & 0.157 & 0.003 & 182.8 & 1.3 & 4 & 1.07 & 0.15 & 4 & 0.56 & 0.10 & 4 & 0.52 & 0.08 \\
\hline 7372 & $\mathrm{AB}$ & .953 & 11 & 0.111 & 0.005 & 276.9 & 3.0 & 4 & 0.24 & 0.13 & 3 & 0.47 & 0.23 & 4 & 0.28 & 0.15 \\
\hline 7372 & $\mathrm{AC}$ & .953 & 12 & 1.794 & 0.004 & 333.5 & 0.2 & 4 & 2.87 & 0.04 & 4 & 2.72 & 0.14 & 4 & 2.46 & 0.02 \\
\hline 7580 & $\mathrm{AB}$ & .953 & 12 & 0.263 & 0.006 & 147.4 & 0.5 & 4 & 0.99 & 0.03 & 4 & 0.91 & 0.07 & 4 & 0.85 & 0.05 \\
\hline 15382 & $\mathrm{AB}$ & .956 & 11 & 0.366 & 0.044 & 273.6 & 4.8 & 3 & 2.10 & 0.24 & 4 & 2.11 & 0.11 & 4 & 2.45 & 0.31 \\
\hline $19233^{(1)}$ & $\mathrm{AB}$ & .953 & 12 & 0.415 & 0.016 & 294.3 & 1.1 & 4 & 2.86 & 0.12 & 4 & 2.85 & 0.07 & 4 & 2.83 & 0.08 \\
\hline 21280 & $\mathrm{AB}$ & .956 & 12 & 0.170 & 0.003 & 232.4 & 2.1 & 4 & 1.15 & 0.15 & 4 & 0.68 & 0.02 & 4 & 0.66 & 0.06 \\
\hline 22550 & $\mathrm{AB}$ & .953 & 12 & 0.210 & 0.003 & 274.1 & 0.5 & 4 & 0.08 & 0.02 & 4 & 0.10 & 0.02 & 4 & 0.13 & 0.02 \\
\hline 22573 & $\mathrm{AB}$ & .953 & 12 & 0.206 & 0.005 & 31.6 & 0.3 & 4 & 0.40 & 0.02 & 4 & 0.29 & 0.03 & 4 & 0.29 & 0.04 \\
\hline 22607 & $\mathrm{AB}$ & .953 & 12 & 0.527 & 0.005 & 228.8 & 0.2 & 4 & 1.25 & 0.02 & 4 & 1.12 & 0.02 & 4 & 1.11 & 0.02 \\
\hline 25037 & $\mathrm{AB}$ & .956 & 8 & 0.177 & 0.006 & 301.7 & 1.3 & - & - & - & 4 & 0.67 & 0.09 & 4 & 0.67 & 0.05 \\
\hline 25119 & $\mathrm{AB}$ & .953 & 12 & 1.225 & 0.002 & 171.4 & 0.2 & 4 & 1.52 & 0.02 & 4 & 1.12 & 0.03 & 4 & 1.07 & 0.02 \\
\hline 27901 & $\mathrm{AB}$ & .953 & 8 & 0.177 & 0.004 & 176.2 & 0.8 & - & - & - & 4 & 1.10 & 0.17 & 4 & 1.06 & 0.04 \\
\hline 29234 & $\mathrm{AB}$ & .953 & 12 & 0.178 & 0.003 & 294.1 & 0.7 & 4 & -0.03 & 0.04 & 4 & 0.09 & 0.08 & 4 & 0.12 & 0.04 \\
\hline 30920 & $\mathrm{AB}$ & .956 & 5 & 0.562 & 0.007 & 339.1 & 0.8 & 5 & 1.63 & 0.04 & - & - & - & - & - & - \\
\hline 30953 & $\mathrm{AB}$ & .956 & 12 & 0.732 & 0.001 & 265.8 & 0.2 & 4 & 0.05 & 0.02 & 4 & 0.07 & 0.02 & 4 & 0.05 & 0.02 \\
\hline 33451 & $\mathrm{AB}$ & .953 & 12 & 0.265 & 0.013 & 185.2 & 0.6 & 4 & 0.29 & 0.03 & 4 & 0.24 & 0.04 & 4 & 0.28 & 0.03 \\
\hline 40167 & $\mathrm{AB}$ & .956 & 1 & 0.859 & - & 78.2 & - & - & - & - & - & - & - & 1 & 0.18 & - \\
\hline 40167 & $\mathrm{~A}, \mathrm{Ca}$ & .956 & 1 & 6.220 & - & 73.2 & - & - & - & - & - & - & - & 1 & 0.56 & - \\
\hline 40167 & $\mathrm{~A}, \mathrm{Cb}$ & .956 & 1 & 6.546 & - & 72.9 & - & - & - & - & - & - & - & 1 & 1.61 & - \\
\hline 41261 & $\mathrm{AB}$ & .953 & 12 & 0.199 & 0.003 & 355.4 & 0.4 & 4 & 0.34 & 0.06 & 4 & 0.30 & 0.02 & 4 & 0.30 & 0.02 \\
\hline 42075 & $\mathrm{AB}$ & .953 & 12 & 0.190 & 0.007 & 96.4 & 1.5 & 4 & 0.99 & 0.16 & 4 & 0.98 & 0.07 & 4 & 0.97 & 0.12 \\
\hline 45170 & $\mathrm{Aa}, \mathrm{Ab}$ & .953 & 12 & 0.117 & 0.007 & 296.1 & 3.5 & 4 & 0.26 & 0.28 & 4 & 0.09 & 0.10 & 4 & 0.03 & 0.03 \\
\hline 46404 & $\mathrm{AB}$ & .956 & 12 & 0.372 & 0.026 & 153.4 & 0.9 & 4 & 1.30 & 0.04 & 4 & 1.62 & 0.03 & 4 & 1.71 & 0.12 \\
\hline 46454 & $\mathrm{AB}$ & .956 & 12 & 0.591 & 0.004 & 86.3 & 0.3 & 4 & 0.64 & 0.02 & 4 & 0.76 & 0.03 & 4 & 0.84 & 0.02 \\
\hline 47479 & $\mathrm{AB}$ & .953 & 12 & 0.161 & 0.004 & 71.2 & 0.8 & 4 & 0.10 & 0.03 & 4 & 0.13 & 0.02 & 4 & 0.13 & 0.02 \\
\hline 53840 & $\mathrm{AB}$ & .953 & 12 & 0.165 & 0.002 & 285.4 & 0.6 & 4 & 0.18 & 0.04 & 4 & 0.25 & 0.04 & 4 & 0.30 & 0.02 \\
\hline 114576 & $\mathrm{AB}$ & .953 & 12 & 0.243 & 0.007 & 113.3 & 0.9 & 4 & 0.23 & 0.02 & 4 & 0.25 & 0.03 & 4 & 0.18 & 0.01 \\
\hline
\end{tabular}

(1) new candidate binary star.

In Table 5, we report some measurements of our selected targets made by other observers. For each target, we indicate the concerned pair in Col. 2, and display our measurements $\left.\rho_{\text {all }}, \theta_{\text {all }}\right)$ in Cols. 3 and 4 , the measurements $\rho_{d}, \theta_{d}$ ) from other sources in Cols. 5 and 6 , and the corresponding position differences in Cols. 7,8 , 9 in polar $\left(\rho_{\text {all }}-\rho_{d}, \theta_{\text {all }}-\theta_{d}\right)$ and Cartesian coordinates $(\Delta$ Pos), with the epoch of observation of those measurements in Col. 7 and their bibliographic reference in Col. 8. The mention "WDS" is used when the data come from the Washington Double Star Catalog (Mason et al., 2012). Note that this comparison is meaningful only when the epoch of the other measurements is close to 2000.95, the epoch of our observations, or when the couples have a very long orbital period.

There is a fair agreement, with $\Delta \mathrm{Pos}<0.1^{\prime \prime}$ in general. The largest discrepancy concerns HIP 27901
(FIN 382). This is also the object having the second largest $O-C$ residual reported in Table 4. Our new measurement of HIP 27901 appears to be inconsistent both with orbits of Cvetkovic (2008) and Hartkopf et al. (2012a). We still think that our measurements are valid and discard an identification error, because they are in close agreement with those made by Hipparcos for the epoch 1991.25 (see Table 5). The situation is not at all clear, the more that some older measurements too were discarded by Cvetkovic (2008) in his orbit computation because they were found to be incompatible with the proposed 32-yr period (e.g. the measurement from 1979). Clearly more observations are needed for this system.

The case of HIP 25037 (STT 105) is also particularly interesting since the companion had not been measured for nearly thirty years and high-angular res- 
Table 3 Relative astrometry obtained by Fourier analysis. In col. 6, the asterisk indicates a possible $180^{\circ}$-ambiguity on $\theta$.

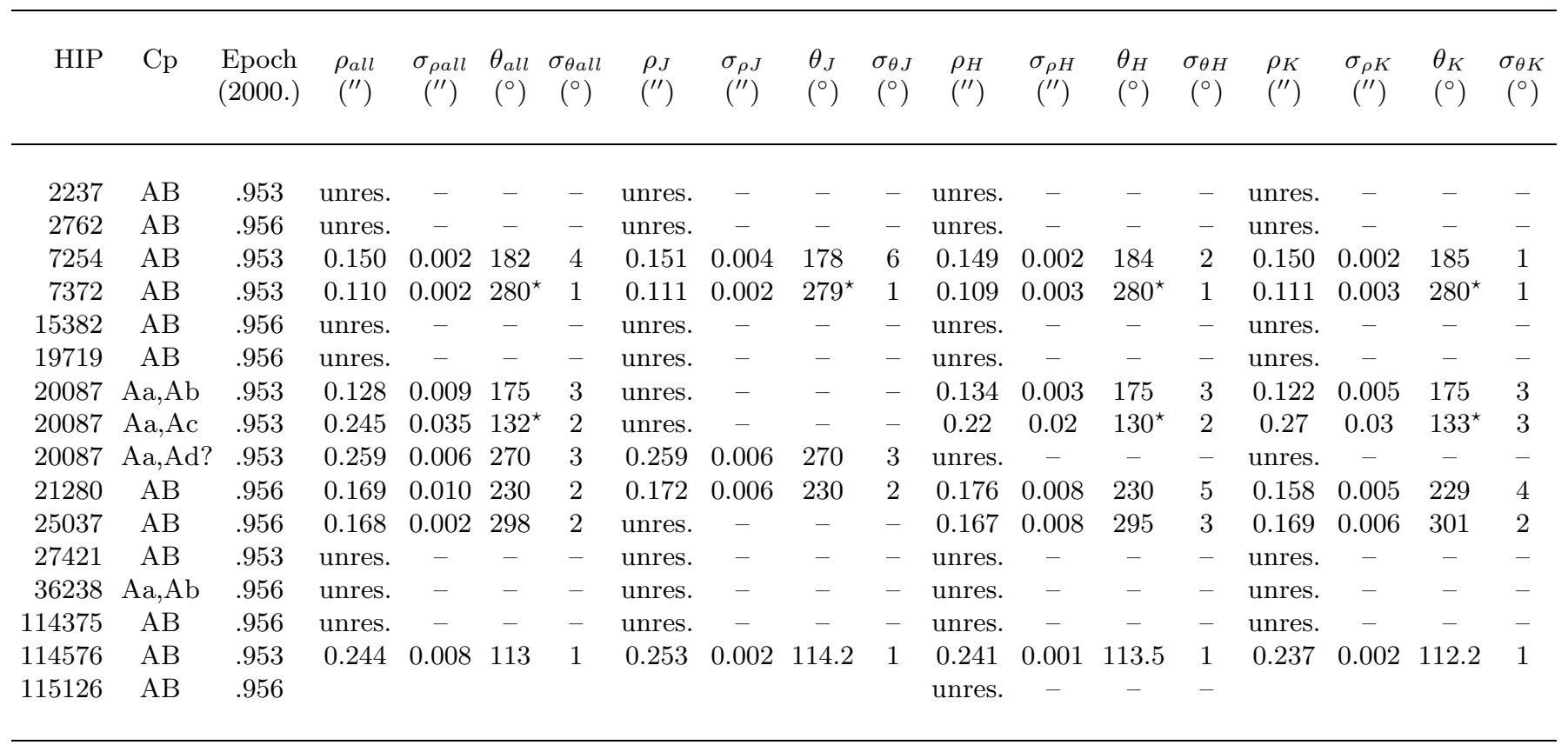

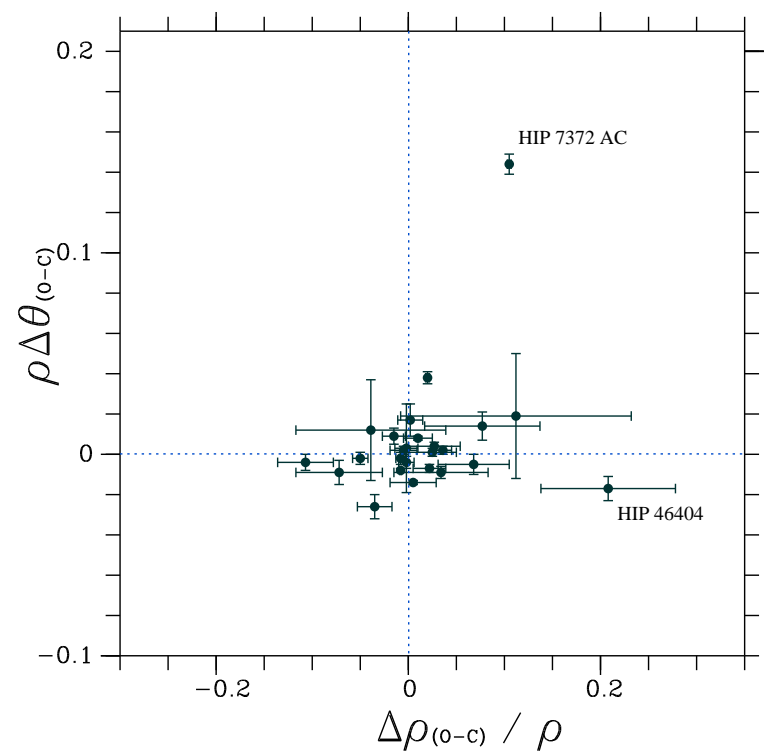

Fig. 4 Illustration of the $(\mathrm{O}-\mathrm{C})$ residuals from Table 4 : $\rho \Delta \theta_{(O-C)}$ versus $\Delta \rho_{(O-C)} / \rho$. The units used here are arcseconds for $\rho$ and $\Delta \rho_{O-C}$, and radians for $\Delta \theta_{O-C}$.

olution data obtained from speckle or AO measurements were deemed necessary. Our measurement is the first direct observation made since 1971 and perfectly matches the model of a linear motion of component A. This target is thus likely to be a (close) optical pair with an apparent closest approach during the interval 1980-1990 (see Fig. 5 where we plotted the apparent

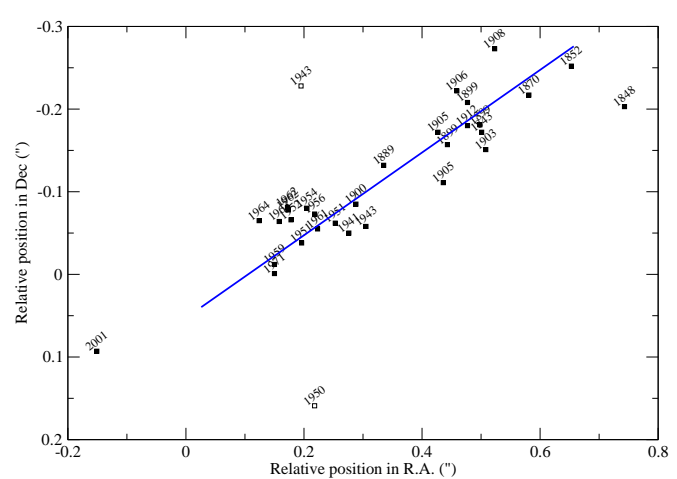

Fig. 5 HIP 25037 (STT 105): relative motion of component B versus component A in the period 1980-1990. In fact $\mathrm{A}$ has a proper motion in the direction of $169^{\circ}$, whereas $\mathrm{B}$ seems still relative to the celestial sphere. North is to the bottom and East to the right.

motion of $\mathrm{B}$ relative to $\mathrm{A}$ ). This (recti)linear motion is also compatible with the proper motion of component A measured by Hipparcos $\left(\mu_{\alpha}(*)=5.67 \mathrm{mas} / \mathrm{yr}\right.$; $\mu_{\delta}=-29.35$ mas $/ y r$ (ESA, 1997), indicating a proper motion in the direction of $169^{\circ}$. It seems that the B star has no significant proper motion and was still relative to the celestial sphere in the period 1980-1990. 
Table 4 Comparison between observations and predicted relative positions.

\begin{tabular}{|c|c|c|c|c|c|c|c|c|c|c|c|c|c|}
\hline $\mathrm{HIP}^{a}$ & $\mathrm{Cp}$ & $\mathrm{N}$ & $\begin{array}{c}\rho_{\text {all }} \\
\left({ }^{\prime \prime}\right)\end{array}$ & $\begin{array}{l}\sigma_{\rho} \\
\left({ }^{\prime \prime}\right)\end{array}$ & $\begin{array}{c}\theta_{\text {all }} \\
\left({ }^{\circ}\right)\end{array}$ & $\begin{array}{l}\sigma_{\theta} \\
\left(^{\circ}\right)\end{array}$ & $\begin{array}{l}\rho_{c} \\
\left({ }^{\prime \prime}\right)\end{array}$ & $\begin{array}{l}\theta_{c} \\
\left({ }^{\circ}\right)\end{array}$ & $\begin{array}{c}\Delta \rho_{(O-C)} \\
\quad\left({ }^{\prime \prime}\right)\end{array}$ & $\begin{array}{c}\Delta \theta_{(O-C)} \\
\quad\left({ }^{\circ}\right)\end{array}$ & $\begin{array}{c}\Delta \operatorname{Pos} \\
\left({ }^{\prime \prime}\right)\end{array}$ & $\begin{array}{c}\Delta \operatorname{Pos} / \rho \\
(\%)\end{array}$ & $\begin{array}{l}\text { Orbit } \\
\text { reference }\end{array}$ \\
\hline 730 & $\mathrm{AB}$ & 11 & 0.281 & 0.022 & 59.6 & 5.0 & 0.292 & 57.1 & -0.011 & 2.5 & 0.016 & 5.9 & Argyle et al. (2002) \\
\hline 2941 & $\mathrm{AB}$ & 12 & 0.554 & 0.003 & 292.5 & 0.4 & 0.558 & 293.2 & -0.004 & -0.7 & 0.008 & 1.4 & Hartkopf et al. (2010) \\
\hline 5165 & $\mathrm{AB}$ & 4 & 0.241 & 0.002 & 238.3 & 0.6 & 0.253 & 238.8 & -0.012 & -0.5 & 0.012 & 5.1 & Alzner et al. (2009) \\
\hline 5842 & $\mathrm{CD}$ & 4 & 0.947 & 0.011 & 275.8 & 0.2 & 0.961 & 275.3 & -0.014 & 0.5 & 0.016 & 1.7 & Ruymaekers (1999) \\
\hline 7372 & $\mathrm{AB}$ & 11 & 0.111 & 0.005 & 276.9 & 3.0 & 0.120 & $281.4^{Q}$ & -0.009 & -4.5 & 0.013 & 11.3 & Mason et al. (1999) \\
\hline 7372 & $\mathrm{AB}$ & 11 & 0.110 & 0.001 & $279.7^{Q}$ & 1. & 0.120 & $281.4^{Q}$ & -0.010 & -1.7 & 0.011 & 9.6 & Mason et al. (1999) \\
\hline 7372 & $\mathrm{AC}$ & 12 & 1.794 & 0.004 & 333.5 & 0.2 & 1.606 & 328.9 & 0.188 & 4.6 & 0.233 & 13.2 & Ruymaekers (1999) \\
\hline 7580 & $\mathrm{AB}$ & 12 & 0.263 & 0.006 & 147.4 & 0.5 & 0.256 & 146.6 & 0.007 & 0.8 & 0.008 & 3.0 & Pourbaix (2000) \\
\hline 15382 & $\mathrm{AB}$ & 11 & 0.366 & 0.044 & 273.6 & 4.8 & 0.325 & $270.6^{Q}$ & 0.041 & 3.0 & 0.045 & 12.4 & Seymour et al. (2002) \\
\hline 20087 & $\mathrm{Aa}, \mathrm{Ab}$ & 8 & 0.128 & 0.009 & 175.0 & 3.0 & 0.112 & $170.1^{Q}$ & 0.016 & 4.9 & 0.019 & 15.1 & Pourbaix $(2000)$ \\
\hline 21280 & $\mathrm{AB}$ & 12 & 0.170 & 0.003 & 232.4 & 2.1 & 0.163 & 232.4 & 0.007 & 0.0 & 0.007 & 4.1 & Cvetkovic \& Ninkovic (2008) \\
\hline 21280 & $\mathrm{AB}$ & 12 & 0.169 & 0.010 & 230.0 & 2. & 0.163 & 232.4 & 0.006 & -2.4 & 0.009 & 5.5 & Cvetkovic \& Ninkovic (2008) \\
\hline 22550 & $\mathrm{AB}$ & 12 & 0.210 & 0.003 & 274.1 & 0.5 & 0.209 & 273.5 & -0.001 & 0.6 & 0.002 & 1.2 & Söderhjelm (1999) \\
\hline 22573 & $\mathrm{AB}$ & 12 & 0.206 & 0.005 & 31.6 & 0.3 & 0.207 & 35.4 & -0.001 & -3.8 & 0.014 & 6.7 & Docobo \& Andrade (2012) \\
\hline 22607 & $\mathrm{AB}$ & 12 & 0.527 & 0.005 & 228.8 & 0.2 & 0.546 & 228.6 & -0.019 & 0.2 & 0.019 & 3.6 & Söderhjelm (1999) \\
\hline 25119 & $\mathrm{AB}$ & 12 & 1.225 & 0.002 & 171.4 & 0.1 & 1.209 & 169.6 & 0.016 & 1.8 & 0.042 & 3.4 & Söderhjelm (1999) \\
\hline 27901 & $\mathrm{AB}$ & 8 & 0.177 & 0.004 & 176.2 & 0.8 & 0.254 & $179.1^{Q}$ & -0.077 & -2.9 & 0.078 & 43.8 & Cvetkovic (2008) \\
\hline$"$ & $"$ & $"$ & $"$ & $"$ & $"$ & $"$ & 0.249 & $212.4^{Q}$ & -0.072 & -36.0 & 0.132 & 74.8 & Hartkopf et al. (2012a) \\
\hline 29234 & $\mathrm{AB}$ & 12 & 0.178 & 0.003 & 294.1 & 0.7 & 0.174 & 296.3 & 0.004 & -2.2 & 0.008 & 4.5 & Hartkopf et al. (1996) \\
\hline 30920 & $\mathrm{AB}$ & 5 & 0.562 & 0.007 & 339.1 & 0.8 & 0.559 & 337.4 & 0.003 & 1.7 & 0.017 & 3.0 & Ségransan et al. (2000) \\
\hline 30953 & $\mathrm{AB}$ & 12 & 0.732 & 0.001 & 265.8 & 0.1 & 0.738 & 266.4 & -0.006 & -0.6 & 0.010 & 1.3 & Hartkopf et al. (2010) \\
\hline 33451 & $\mathrm{AB}$ & 12 & 0.265 & 0.013 & 185.2 & 0.6 & 0.256 & 187.2 & 0.009 & -2.0 & 0.013 & 4.9 & Docobo et al. (2009) \\
\hline 40167 & $\mathrm{AB}$ & 1 & 0.859 & - & 78.2 & - & 0.861 & 78.4 & -0.002 & -0.2 & 0.004 & 0.4 & Ruymaekers (1999) \\
\hline 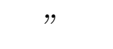 & & $"$ & & $"$ & 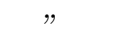 & $"$ & 0.854 & 78.9 & 0.005 & -0.7 & 0.012 & 1.4 & Mason et al. (2006) \\
\hline 41261 & $\mathrm{AB}$ & 12 & 0.199 & 0.003 & 355.4 & 0.4 & 0.197 & 353.0 & 0.002 & 2.4 & 0.009 & 4.3 & Hartkopf et al. (2012a) \\
\hline 42075 & $\mathrm{AB}$ & 12 & 0.190 & 0.007 & 96.4 & 1.5 & 0.174 & 97.9 & 0.016 & -1.5 & 0.017 & 8.8 & Docobo \& Andrade (2012) \\
\hline 45170 & $\mathrm{Aa}, \mathrm{Ab}$ & 12 & 0.117 & 0.007 & 296.1 & 3.5 & 0.117 & $294.8^{Q}$ & 0.000 & 1.3 & 0.003 & 2.3 & Mason et al. (1996) \\
\hline$"$ & $"$ & $"$ & . & $"$ & $"$ & $"$ & 0.108 & $289.2^{Q}$ & 0.009 & 6.9 & 0.017 & 14.3 & Ruymaekers (1999) \\
\hline 46404 & $\mathrm{AB}$ & 12 & 0.372 & 0.026 & 153.4 & 0.9 & 0.295 & 156.0 & 0.077 & -2.6 & 0.079 & 21.2 & Söderhjelm (1999) \\
\hline 46454 & $\mathrm{AB}$ & 12 & 0.591 & 0.004 & 86.3 & 0.3 & 0.592 & 86.7 & -0.001 & -0.4 & 0.004 & 0.7 & Muterspaugh et al. (2010) \\
\hline 47479 & $\mathrm{AB}$ & 12 & 0.161 & 0.004 & 71.2 & 0.8 & 0.157 & 70.8 & 0.004 & 0.4 & 0.004 & 2.6 & Söderhjelm (1999) \\
\hline 114576 & $\mathrm{AB}$ & 12 & 0.243 & 0.007 & 113.3 & 0.9 & 0.240 & 114.3 & 0.004 & -1.0 & 0.005 & 2.1 & Pourbaix (2000) \\
\hline 114576 & $\mathrm{AB}$ & 12 & 0.244 & 0.008 & 113.0 & 1. & 0.240 & 114.3 & 0.004 & -1.3 & 0.007 & 2.8 & Pourbaix (2000) \\
\hline
\end{tabular}

${ }^{a}:$ The HIP identifier is in italic font when the corresponding measurement was derived by Fourier analysis.

$Q$ : This symbol is used when a quadrant correction of $180^{\circ}$ has been applied.

\subsection{Analysis of photometric measurements}

Another important piece of information derived from Moffat profile fitting is the differential photometry between the two stellar components The values we obtained are reported in Table 2, in Cols. 10, 13 and 16, for the filters $J, H$ and $K$, respectively, with the corresponding errors in Cols. 11, 14 and 17. As shown in Figs. 6 and 7, we were able to measure large differences of magnitudes, up to 3 mag. The average rms photometric errors for $\sigma_{\Delta m_{J}}, \sigma_{\Delta m_{H}}$, and $\sigma_{\Delta m_{K}}$ are $0.06,0.06$ and 0.07 mag, respectively.

We next made use of archived information from the 2MASS Catalogue (a survey operated by IPAC Infrared Science Archive (IRSA), Caltech/JPL) to extract to- tal magnitudes in the near-infrared passbands for the observed systems. We also searched through the bibliographic data base of SIMBAD (CDS, Strasbourg). Table 6 lists the total magnitudes in the $J-, H$ - and $K$-bands with their respective errors collected in this way. The 2MASS JHK photometric system is very close to the ADONIS ESO JHK filter system we used (see Bouchet, 1991, and Bessel, 2005).

In combination with the measured differential magnitudes (see Table 2), we then derived the component magnitudes from the above-mentioned data for $27 \mathrm{bi}-$ nary and multiple systems. This enabled us to also derive the component colours for most of these systems. These results are reported in Table 7 and plotted in Fig. 8. In this figure, we also plotted the average val- 
Table 5 Comparison between some of our measurements $\left(\rho_{\text {all }}, \theta_{\text {all }}\right)$ made in 2000.95 and other published data $\left(\rho_{d}, \theta_{d}\right)$.

\begin{tabular}{|c|c|c|c|c|c|c|c|c|c|c|}
\hline $\mathrm{HIP}^{a}$ & $\mathrm{Cp}$ & $\begin{array}{c}\rho_{\text {all }} \\
\left({ }^{\prime \prime}\right)\end{array}$ & $\begin{array}{c}\theta_{\text {all }} \\
\left({ }^{\circ}\right)\end{array}$ & $\begin{array}{l}\rho_{d} \\
\left({ }^{\prime \prime}\right)\end{array}$ & $\begin{array}{l}\theta_{d} \\
\left(^{\circ}\right)\end{array}$ & $\begin{array}{c}\rho_{a l l}-\rho_{d} \\
\left({ }^{\prime \prime}\right)\end{array}$ & $\begin{array}{c}\theta_{a l l}-\theta_{d} \\
\quad\left({ }^{\circ}\right)\end{array}$ & $\begin{array}{c}\Delta \text { Pos } \\
\left({ }^{\prime \prime}\right)\end{array}$ & Epoch & Reference \\
\hline 7254 & $\mathrm{AB}$ & 0.157 & 182.8 & 0.1 & 185. & 0.057 & -2.2 & 0.057 & 1991.25 & ESA, 1997 \\
\hline 7254 & $\mathrm{AB}$ & 0.150 & 182.0 & 0.1 & 185. & 0.050 & -3.0 & 0.050 & 1991.25 & ESA, 1997 \\
\hline 27901 & $\mathrm{AB}$ & 0.177 & 176.2 & 0.165 & 179. & 0.012 & -2.8 & 0.015 & 1991.25 & $\mathrm{ESA}, 1997$ \\
\hline$"$ & $"$ & $"$ & $"$ & 0.1 & 142. & 0.077 & 34.2 & 0.110 & 1993.10 & Hartkopf et al., 2000 \\
\hline 40167 & $\mathrm{AC}$ & 6.22 & 73.2 & 6.17 & 73.5 & 0.050 & -0.3 & 0.062 & 2001.24 & Mason et al., 2002 \\
\hline 40167 & $\mathrm{Ca}, \mathrm{Cb}$ & 0.327 & 67.7 & 0.317 & 84.5 & 0.010 & -16.8 & 0.096 & 2000.14 & Hutchings et al., 2000 \\
\hline$"$ & $"$ & , & $\mathrm{se}$ & 0.338 & 62.1 & -0.011 & 5.6 & 0.034 & 2001.27 & Balega et al., 2006 \\
\hline 53840 & $\mathrm{AB}$ & 0.165 & 285.4 & 0.1 & $304 .^{Q}$ & 0.065 & 18.7 & 0.077 & 1994.31 & Hartkopf et al., 1996b \\
\hline
\end{tabular}

${ }^{a}$ : For a given identifier in italic font, the measurement was derived by Fourier analysis.

$Q$ : This symbol is used when a quadrant correction of $180^{\circ}$ has been applied.

Table 6 Infrared (system) photometry from 2MASS survey and other bibliographic sources

\begin{tabular}{|c|c|c|c|c|}
\hline HIP & $\begin{array}{c}\mathrm{J}_{t o t} \\
(\mathrm{mag})\end{array}$ & $\begin{array}{c}\mathrm{H}_{t o t} \\
(\mathrm{mag})\end{array}$ & $\begin{array}{c}\mathrm{K}_{t o t} \\
(\mathrm{mag})\end{array}$ & Comments \\
\hline 730 & - & $4.590 \pm 0.075$ & $4.408 \pm 0.029$ & 2MASS \\
\hline 2941 & 4.08 & 3.78 & 3.73 & HD 3443, Alonso et al. (1994) \\
\hline 5165 & $1.831 \pm 0.020$ & $1.390 \pm 0.020$ & $1.286 \pm 0.020$ & Bouchet et al. (1991) \\
\hline 5842 & $5.588 \pm 0.019$ & $5.066 \pm 0.021$ & $4.980 \pm 0.013$ & NLTT 4206, 2MASS \\
\hline 7254 & $6.388 \pm 0.017$ & $5.992 \pm 0.027$ & $5.894 \pm 0.005$ & NLTT 5201, 2MASS \\
\hline 7372 & $5.340 \pm 0.015$ & $4.973 \pm 0.075$ & $4.690 \pm 0.011$ & GJ 60 ABC, 2MASS \\
\hline 7580 & $5.211 \pm 0.011$ & $5.024 \pm 0.025$ & $4.893 \pm 0.011$ & NLTT 5442, 2MASS \\
\hline 15382 & $3.405 \pm 0.284$ & $2.844 \pm 0.254$ & $2.741 \pm 0.270$ & 2MASS \\
\hline 19233 & $5.220 \pm 0.010$ & $4.905 \pm 0.007$ & $4.824 \pm 0.008$ & Bouchet et al. (1991) \\
\hline 21280 & $6.989 \pm 0.019$ & $6.576 \pm 0.019$ & $6.465 \pm 0.019$ & 2MASS, vA 727 Carney (1982) \\
\hline 22550 & $5.710 \pm 0.025$ & $5.523 \pm 0.033$ & $5.436 \pm 0.007$ & 2MASS \\
\hline 22573 & $5.604 \pm 0.015$ & $5.553 \pm 0.037$ & $5.520 \pm 0.015$ & 2MASS \\
\hline 22607 & $5.235 \pm 0.023$ & $5.030 \pm 0.011$ & $4.941 \pm 0.003$ & 2MASS \\
\hline 25037 & $7.318 \pm 0.011$ & $7.235 \pm 0.019$ & $7.172 \pm 0.015$ & 2MASS \\
\hline 25119 & $5.940 \pm 0.011$ & $5.456 \pm 0.033$ & $5.302 \pm 0.015$ & 2MASS \\
\hline 27901 & $5.606 \pm 0.017$ & $5.439 \pm 0.023$ & $5.381 \pm 0.011$ & 2MASS \\
\hline 29234 & $4.974 \pm 0.272$ & $4.653 \pm 0.236$ & $4.586 \pm 0.007$ & 2MASS \\
\hline 30920 & $6.376 \pm 0.015$ & $5.754 \pm 0.031$ & $5.486 \pm 0.005$ & V577 Mon, 2MASS \\
\hline 30953 & $4.774 \pm 0.234$ & $4.477 \pm 0.240$ & $4.334 \pm 0.016$ & 2MASS \\
\hline 33451 & $5.379 \pm 0.013$ & $5.171 \pm 0.019$ & $5.090 \pm 0.015$ & 2MASS \\
\hline 41261 & $6.262 \pm 0.015$ & $5.902 \pm 0.023$ & $5.790 \pm 0.017$ & 2MASS \\
\hline 42075 & $5.328 \pm 0.262$ & $4.748 \pm 0.268$ & $4.460 \pm 0.019$ & 2MASS \\
\hline 45170 & $5.121 \pm 0.013$ & $4.773 \pm 0.033$ & $4.664 \pm 0.009$ & LHS 2114, 2MASS \\
\hline 46404 & $4.125 \pm 0.007$ & $3.815 \pm 0.007$ & $3.760 \pm 0.014$ & HD 81809, Alonso et al. (1994) \\
\hline 46454 & $4.656 \pm 0.236$ & $4.262 \pm 0.214$ & $3.946 \pm 0.033$ & $2 \mathrm{MASS}$ \\
\hline 47479 & $5.369 \pm 0.312$ & $5.055 \pm 0.338$ & $4.798 \pm 0.013$ & GJ 9305, 2MASS \\
\hline 53840 & $5.764 \pm 0.007$ & $5.471 \pm 0.021$ & $5.396 \pm 0.013$ & NLTT 26003, 2MASS \\
\hline 114576 & $6.546 \pm 0.013$ & $6.308 \pm 0.015$ & $6.184 \pm 0.009$ & 2MASS \\
\hline
\end{tabular}




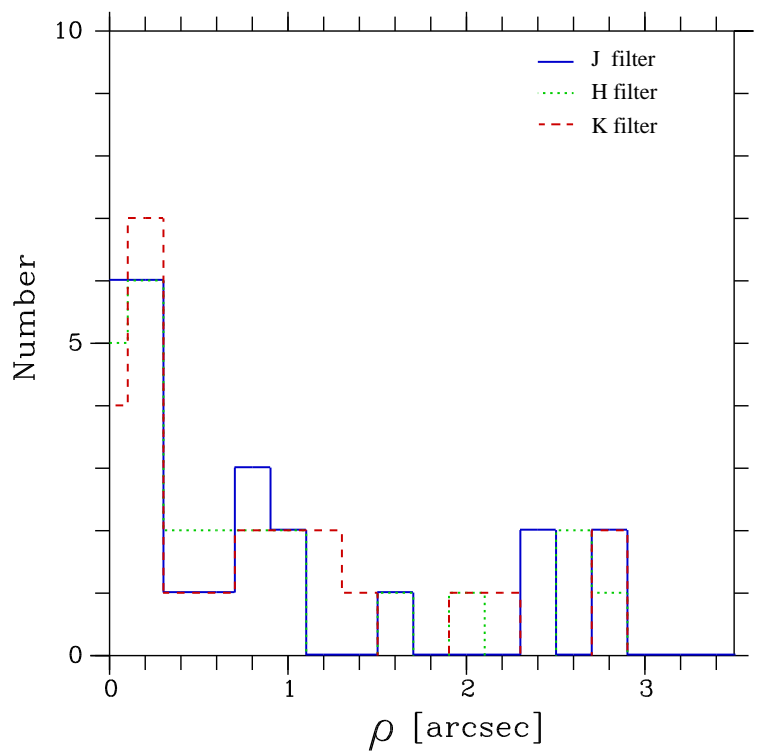

Fig. 6 Distributions of the magnitude differences between the two components $\Delta m_{J}$ (blue solid line), $\Delta m_{H}$ (green dotted line) and $\Delta m_{K}$ (red dashed line), reported in Table 2 .

ues obtained on sample of about 200 standard stars by other observers at ESO (Bouchet et al. (1991). Those standard stars were apparently single stars. Some of the spectral types are indicated close to the corresponding data points.

The individual stellar components have nearinfrared colour indices rather well grouped along the line formed by the ESO standard stars. The dispersion is rather small. The only possible outlier is the B component of HIP $15382 \mathrm{AB}$ (whose primary is indicated to be a K0-III star), which has an $H-K$ index slightly smaller from the other stars of our sample. However our sample is very small which makes any conclusion on this object too premature.

\section{Conclusion}

We obtained relative astrometric and differential photometric data for a sample of nearby southern orbital binaries for which high-quality Hipparcos parallaxes and supposed well-known orbits were already available, but for which accurate component magnitudes and/or colour indices were lacking. This lack of information may explain why some of these binaries did not fit well the empirical mean mass-luminosity relation first derived with the new Hipparcos parallaxes (Lampens et al. 1997). For about 30 binary systems of the sample, we additionally derived the component magnitudes and colour indices by combining existing total $J_{-}, H$-, and $K$ - magnitudes with our measurements of differential colours in the near-infrared $J$-, $H$-, $K$-passbands. Among these 30 observed systems,

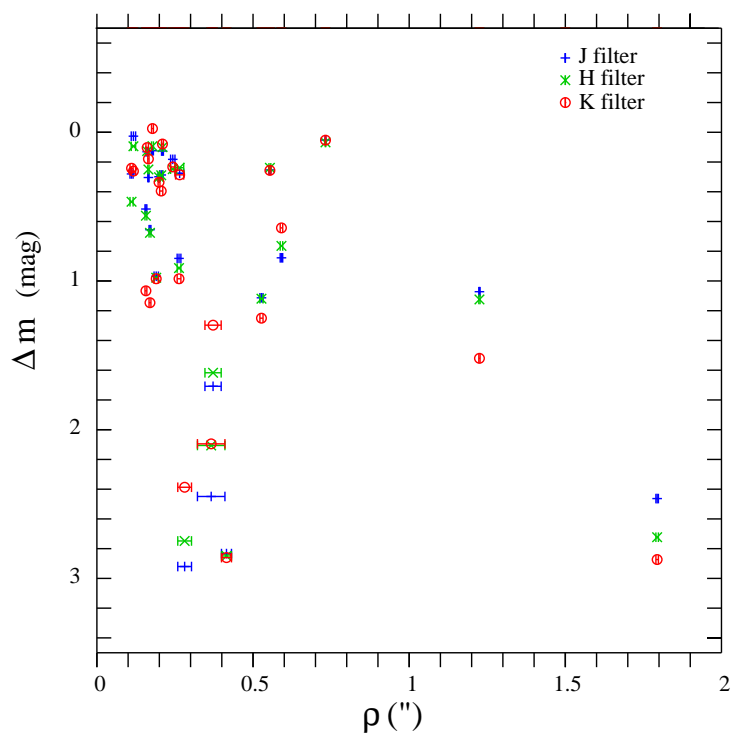

Fig. 7 Magnitude difference between the two components plotted versus their angular separation. Those parameters were obtained by fitting Moffat profiles to the AO images (see Table 2).

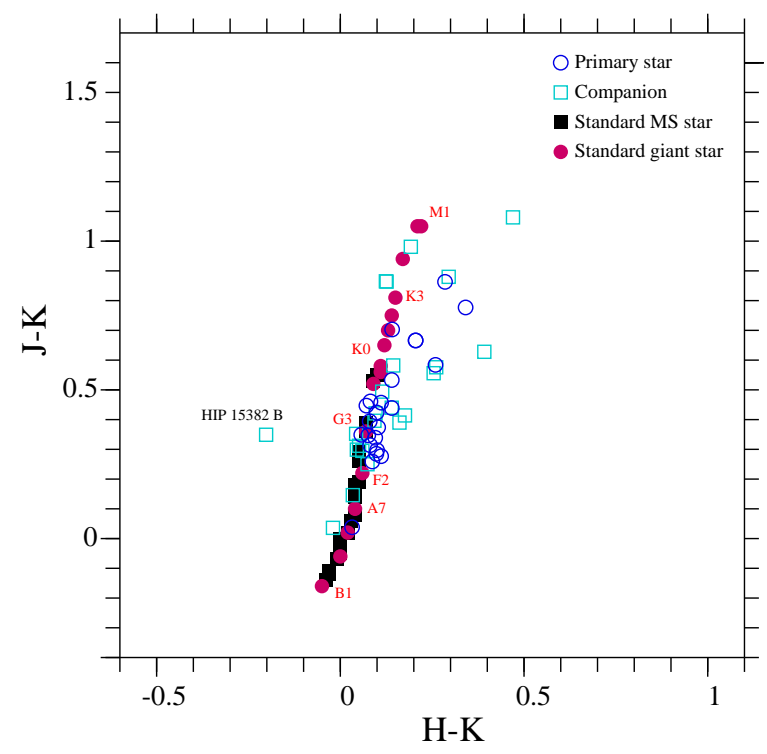

Fig. 8 Near-infrared colour indices of the individual stellar components derived from Table 7. Used symbols are blue open circles for the primary components and blue open squares for the secondary components. Standard stars (from Bouchet et al., 1991) are plotted as black filled squares for main sequence stars and red filled circles for giant stars. 
Table 7 Derived infrared component magnitudes and colours.

\begin{tabular}{|c|c|c|c|c|c|c|c|c|c|c|c|c|c|c|}
\hline HIP & $\mathrm{Cp}$ & $\mathrm{N}_{t o t}$ & $\begin{array}{c}\mathrm{J}_{1} \\
(\mathrm{mag})\end{array}$ & $\begin{array}{c}\mathrm{J}_{2} \\
(\mathrm{mag})\end{array}$ & $\begin{array}{c}\mathrm{H}_{1} \\
(\mathrm{mag})\end{array}$ & $\begin{array}{c}\mathrm{H}_{2} \\
(\mathrm{mag})\end{array}$ & $\begin{array}{c}\mathrm{K}_{1} \\
(\mathrm{mag})\end{array}$ & $\begin{array}{c}\mathrm{K}_{2} \\
(\mathrm{mag})\end{array}$ & $\begin{array}{c}(\mathrm{J}-\mathrm{H})_{1} \\
(\mathrm{mag})\end{array}$ & $\begin{array}{c}(\mathrm{J}-\mathrm{K})_{1} \\
(\mathrm{mag})\end{array}$ & $\begin{array}{c}(\mathrm{H}-\mathrm{K})_{1} \\
(\mathrm{mag})\end{array}$ & $\begin{array}{c}(\mathrm{J}-\mathrm{H})_{2} \\
(\mathrm{mag})\end{array}$ & $\begin{array}{c}(\mathrm{J}-\mathrm{K})_{2} \\
(\mathrm{mag})\end{array}$ & $\begin{array}{c}(\mathrm{H}-\mathrm{K})_{2} \\
\quad(\mathrm{mag})\end{array}$ \\
\hline 730 & $\mathrm{AB}$ & 11 & - & - & 4.67 & 7.42 & 4.48 & 7.40 & - & - & 0.19 & - & - & 0.02 \\
\hline 2941 & $\mathrm{AB}$ & 12 & 4.71 & 4.97 & 4.42 & 4.66 & 4.36 & 4.62 & 0.29 & 0.35 & 0.06 & 0.31 & 0.35 & 0.04 \\
\hline 7254 & $\mathrm{AB}$ & 12 & 6.73 & 7.80 & 6.50 & 7.06 & 6.42 & 6.94 & 0.23 & 0.31 & 0.08 & 0.74 & 0.86 & 0.13 \\
\hline 7372 & $\mathrm{AB}$ & 11 & 5.98 & 6.22 & 5.52 & 5.98 & 5.31 & 5.60 & 0.46 & 0.67 & 0.21 & 0.24 & 0.63 & 0.40 \\
\hline 7372 & $\mathrm{AC}$ & 12 & 5.98 & 8.85 & 5.52 & 8.24 & 5.31 & 7.77 & 0.46 & 0.67 & 0.21 & 0.61 & 1.08 & 0.47 \\
\hline 7580 & $\mathrm{AB}$ & 12 & 5.58 & 6.56 & 5.41 & 6.33 & 5.30 & 6.15 & 0.17 & 0.28 & 0.11 & 0.24 & 0.41 & 0.18 \\
\hline 15382 & $\mathrm{AB}$ & 11 & 3.55 & 5.65 & 2.99 & 5.10 & 2.85 & 5.30 & 0.56 & 0.70 & 0.14 & 0.55 & 0.35 & -0.20 \\
\hline 19233 & $\mathrm{AB}$ & 12 & 5.30 & 8.16 & 4.98 & 7.83 & 4.90 & 7.73 & 0.31 & 0.40 & 0.08 & 0.33 & 0.42 & 0.10 \\
\hline 21280 & $\mathrm{AB}$ & 12 & 7.31 & 8.46 & 7.04 & 7.72 & 6.94 & 7.59 & 0.27 & 0.37 & 0.10 & 0.74 & 0.87 & 0.12 \\
\hline 22550 & $\mathrm{AB}$ & 12 & 6.42 & 6.50 & 6.23 & 6.33 & 6.13 & 6.25 & 0.20 & 0.30 & 0.10 & 0.18 & 0.25 & 0.07 \\
\hline 22573 & $\mathrm{AB}$ & 12 & 6.18 & 6.57 & 6.17 & 6.46 & 6.14 & 6.43 & 0.01 & 0.04 & 0.03 & 0.11 & 0.15 & 0.03 \\
\hline 22607 & $\mathrm{AB}$ & 12 & 5.53 & 6.78 & 5.36 & 6.48 & 5.27 & 6.39 & 0.17 & 0.26 & 0.09 & 0.30 & 0.40 & 0.09 \\
\hline 25037 & $\mathrm{AB}$ & 8 & - & - & 7.70 & 8.37 & 7.64 & 8.31 & - & - & 0.06 & - & - & 0.06 \\
\hline 25119 & $\mathrm{AB}$ & 12 & 6.18 & 7.70 & 5.79 & 6.91 & 5.65 & 6.72 & 0.39 & 0.53 & 0.14 & 0.79 & 0.98 & 0.19 \\
\hline 27901 & $\mathrm{AB}$ & 8 & - & - & 5.78 & 6.88 & 5.73 & 6.78 & - & - & 0.05 & - & - & 0.09 \\
\hline 29234 & $\mathrm{AB}$ & 12 & 5.74 & 5.71 & 5.36 & 5.45 & 5.28 & 5.40 & 0.38 & 0.46 & 0.08 & 0.26 & 0.31 & 0.05 \\
\hline 30920 & $\mathrm{AB}$ & 5 & 6.60 & 8.22 & - & - & - & - & - & - & - & - & - & - \\
\hline 30953 & $\mathrm{AB}$ & 12 & 5.50 & 5.55 & 5.20 & 5.25 & 5.06 & 5.11 & 0.30 & 0.44 & 0.14 & 0.30 & 0.44 & 0.14 \\
\hline 33451 & $\mathrm{AB}$ & 12 & 6.00 & 6.28 & 5.81 & 6.05 & 5.71 & 5.99 & 0.19 & 0.29 & 0.10 & 0.24 & 0.30 & 0.06 \\
\hline 41261 & $\mathrm{AB}$ & 12 & 6.86 & 7.20 & 6.51 & 6.82 & 6.40 & 6.70 & 0.35 & 0.46 & 0.11 & 0.38 & 0.49 & 0.11 \\
\hline 42075 & $\mathrm{AB}$ & 12 & 5.70 & 6.68 & 5.12 & 6.10 & 4.83 & 5.80 & 0.58 & 0.86 & 0.29 & 0.59 & 0.88 & 0.30 \\
\hline 45170 & $\mathrm{Aa}$ & 12 & 5.75 & 6.01 & 5.48 & 5.57 & 5.40 & 5.43 & 0.27 & 0.35 & 0.08 & 0.44 & 0.58 & 0.14 \\
\hline 46404 & $\mathrm{AB}$ & 12 & 4.41 & 5.71 & 4.04 & 5.65 & 3.97 & 5.67 & 0.38 & 0.45 & 0.07 & 0.06 & 0.04 & -0.02 \\
\hline 46454 & $\mathrm{AB}$ & 12 & 5.13 & 5.78 & 4.70 & 5.46 & 4.36 & 5.20 & 0.44 & 0.78 & 0.34 & 0.32 & 0.58 & 0.26 \\
\hline 47479 & $\mathrm{AB}$ & 12 & 6.07 & 6.17 & 5.75 & 5.87 & 5.49 & 5.62 & 0.33 & 0.58 & 0.26 & 0.30 & 0.56 & 0.25 \\
\hline 53840 & $\mathrm{AB}$ & 12 & 6.43 & 6.61 & 6.11 & 6.36 & 6.01 & 6.31 & 0.33 & 0.42 & 0.10 & 0.25 & 0.30 & 0.05 \\
\hline 114576 & $\mathrm{AB}$ & 12 & 7.19 & 7.42 & 6.94 & 7.19 & 6.85 & 7.03 & 0.24 & 0.34 & 0.10 & 0.23 & 0.39 & 0.16 \\
\hline
\end{tabular}

seven targets pertain to the list of systems used by Xia \& Fu (2010), though sometimes without information on the secondary component. We thus provide original data for the secondary (in casu tertiary) components of HIP 2941, HIP 7372, HIP 20087 and HIP 40167.

Our study took advantage of Adaptive Optics observations on a large telescope, that allowed us to derive relative astrometric and differential photometric measurements of the individual components of close binaries. We explained in detail the data reduction procedure that we used, in particular the Moffat profile fitting and the Fourier analysis. The Moffat profile method was very efficient in deriving accurate position and differential magnitude measurements. Fourier analysis was a useful complement for very close binaries, but a major problem was the difficulty to estimate accurately the PSF, since this PSF could not be calibrated simultaneously with the observations of the targets.

Based on a study of the astrometric $O-C$ residuals, we concluded that the orbits of HIP $7372 \mathrm{AC}$ and HIP 46404 as well as those of HIP 21280 and HIP 41261 will need revision in the near future. The measurement we obtained for HIP 27901 is incompatible with the recently proposed orbit(s) and some previous measurements. However, it fits well the Hipparcos data. We suggest that this system needs more follow-up data before any reliable conclusion on its orbit can be drawn. For HIP 25037 AB, we showed that component A follows a rectilinear path on the sky which is also compatible with its proper motion. The system is an optical visual double star.

Fourier analysis furthermore revealed the presence of possibly two new components around HIP 20087, both of which are very close to the diffraction limit of the telescope. We also found another possible new binary star, HIP 19233, with a large magnitude difference. Those two possible serendipitous discoveries need confirming observations.

The newly presented photometric measurements show that most of the stellar components have nearinfrared colour indices comparable to those of normal (apparently single) standard stars. 


\section{Appendix: computation of the gradient of the Moffat profile}

In this appendix, we derive the gradient of our model (see Eq. 4.1 presented in Sect. 4):

$$
I(x, y)=\sum_{m=1,2} I_{m}(x, y)+n_{0}+n_{1} x+n_{2} y+n_{3} x y
$$

with

$$
I_{m}(x, y)=\frac{h_{m}}{\left[1+\left(b r_{m}\right)^{2}\right]^{q}}
$$

The results are reported in Table 8.

The function $I_{m}(x, y)$ can be also written as:

$I_{m}(x, y)=h_{m} \exp \left(-q \log A_{m}\right) \quad$ with $\quad A_{m}=1+\left(b r_{m}\right)^{2}$

It comes:

$$
\frac{\partial I_{m}(x, y)}{\partial h_{m}}=\exp \left(-q \log A_{m}\right)=\frac{I_{m}(x, y)}{h_{m}}
$$

and:

$$
\frac{\partial I_{m}(x, y)}{\partial q}=-\left(\log A_{m}\right) I_{m}(x, y)
$$

Since $\partial A_{m}(x, y) / \partial b=2 r_{m}^{2} b$, it comes:

$$
\frac{\partial I_{m}(x, y)}{\partial b}=-q I_{m}(x, y) \frac{1}{A_{m}}\left(2 r_{m}^{2} b\right)
$$

This can also be written as:

$\frac{\partial I_{m}(x, y)}{\partial b}=-B_{m} r_{m} \quad$ with: $\quad B_{m}=\frac{2 I_{m}(x, y) q r_{m} b}{A_{m}}$

Let us now consider the parameters $e$ and $k$ that are used in the expression of $r_{m}$. Since $\partial A_{m}(x, y) / \partial r_{m}=$ $2 r_{m} b^{2}$, we deduce:

$$
\frac{\partial I_{m}(x, y)}{\partial e}=-\frac{q I_{m}(x, y)\left(2 r_{m} b^{2}\right)}{A_{m}} \frac{\partial r_{m}}{\partial e}=-B_{m} b \frac{\partial r_{m}}{\partial e}
$$

and also:

$$
\frac{\partial I_{m}(x, y)}{\partial k}=-B_{m} b \frac{\partial r_{m}}{\partial k}
$$

Let us recall the expression (4.1) of Sect. 4:

$r_{m}^{2}=\left(x-x_{m}\right)^{2}+e\left(x-x_{m}\right)\left(y-y_{m}\right)+(1+k)\left(y-y_{m}\right)^{2}$

Let us differentiate with respect to $e$ and $k$ :

$$
2 r_{m} \partial r_{m}=\left(x-x_{m}\right)\left(y-y_{m}\right) \partial e+\left(y-y_{m}\right)^{2} \partial k
$$

It comes:

$$
\frac{\partial r_{m}}{\partial e}=\frac{\left(x-x_{m}\right)\left(y-y_{m}\right)}{2 r_{m}}
$$

and

$$
\frac{\partial r_{m}}{\partial k}=\frac{\left(y-y_{m}\right)^{2}}{2 r_{m}}
$$

When substituting in (1) and (2), we obtain:

$$
\frac{\partial I_{m}(x, y)}{\partial e}=-\frac{B_{m} b}{2 r_{m}}\left(x-x_{m}\right)\left(y-y_{m}\right)
$$

and

$$
\frac{\partial I_{m}(x, y)}{\partial k}=-\frac{B_{m} b}{2 r_{m}}\left(y-y_{m}\right)^{2}
$$

Let us now consider the last parameters, which are $x_{m}$ and $y_{m}$. The partial derivative of $I_{m}(x, y)$ relative to those variables are equal to:

$$
\frac{\partial I_{m}(x, y)}{\partial x_{m}}=-B_{m} b \frac{\partial r_{m}}{\partial x_{m}}
$$

and

$$
\frac{\partial I_{m}(x, y)}{\partial y_{m}}=-B_{m} b \frac{\partial r_{m}}{\partial y_{m}}
$$

To obtain the expression of the partial derivatives of $r_{m}$ relative to $x_{m}$ and $y_{m}$, let us develop the expression (3):

$$
\begin{aligned}
r_{m}^{2}= & {\left[x^{2}+e x y+(1+k) y_{m}^{2}\right]-x_{m}(2 x+e y) } \\
& +x_{m}^{2}+e x_{m} y_{m}-y_{m}[x e+2 y(1+k)] \\
& +y_{m}^{2}(1+k)
\end{aligned}
$$

Let us differentiate $(6)$ with respect to $x_{m}$ :

$$
2 r_{m} \partial r_{m}=-(2 x+e y) \partial x_{m}+2 x_{m} \partial x_{m}+e y_{m} \partial x_{m}
$$

It comes:

$$
\frac{\partial r_{m}}{\partial x_{m}}=\frac{-2 x-e y+2 x_{m}+e y_{m}}{2 r_{m}}
$$

After substitution in (5), we finally obtain:

$$
\frac{\partial I_{m}(x, y)}{\partial x_{m}}=-\frac{B_{m} b}{2 r_{m}}\left(-2 x-e y+2 x_{m}+e y_{m}\right)
$$

Similarly, when differentiating the expression (6) with respect to $y_{m}$, we obtain:

$$
\begin{aligned}
2 r_{m} \partial r_{m}= & -[e x+2 y(1+k)] \partial y_{m} \\
& +2 y_{m}(1+k) \partial y_{m}+e x_{m} \partial y_{m}
\end{aligned}
$$

Hence:

$$
\frac{\partial r_{m}}{\partial y_{m}}=\frac{-e x-2 y(1+k)+2 y_{m}(1+k)+e x_{m}}{2 r_{m}}
$$

Let us substitute in (5):

$\frac{\partial I_{m}(x, y)}{\partial y_{m}}=-\frac{B_{m} b}{2 r_{m}}\left[-e x-2 y(1+k)+2 y_{m}(1+k)+e x_{m}\right]$

All the gradient components computed in this appendix are reported in Table 8 .

\section{Acknowledgements}

We thank the ESO staff in Chile for their technical support during the observations. This work has made use of the following data base facilities: 2MASS/IPAC Infrared Science Archive (IRSA), Caltech/JPL; SIMBAD, CDS, Strasbourg; the Washington Double Star Catalog (WDS) maintained at the U.S. Naval Observatory; and the Catalogue of Components of Double and Multiple Stars (CCDM) (Dommanget J. \& Nys O., 2002). We are very grateful to the staff of those scientific institutes. Finally, we would like to thank the referee, W.I. Hartkopf (U.S. Naval Obs.), for his useful comments. 
Table 8 Expression of the components of the gradient of $I_{m}(x, y)$, as a function of the parameters $A_{m}=1+\left(b r_{m}\right)^{2}$ and $B_{m}=2 I_{m}(x, y) q r_{m} b / A_{m}$. The symbol log designates the Napierian logarithm.

$$
\begin{aligned}
\frac{\partial I(x, y)}{\partial n_{0}} & =1 \\
\frac{\partial I(x, y)}{\partial n_{1}} & =x \\
\frac{\partial I(x, y)}{\partial n_{2}} & =y \\
\frac{\partial I(x, y)}{\partial n_{3}} & =x y \\
\frac{\partial I_{m}(x, y)}{\partial h_{m}} & =\frac{I_{m}(x, y)}{h_{m}} \\
\frac{\partial I_{m}(x, y)}{\partial b} & =-B_{m} r_{m} \\
\frac{\partial I_{m}(x, y)}{\partial q} & =-\left(\log A_{m}\right) I_{m}(x, y) \\
\frac{\partial I_{m}(x, y)}{\partial e} & =-\frac{B_{m} b}{2 r_{m}}\left(x-x_{m}\right)\left(y-y_{m}\right) \\
\frac{\partial I_{m}(x, y)}{\partial k} & =-\frac{B_{m} b}{2 r_{m}}\left(y-y_{m}\right)^{2} \\
\frac{\partial I_{m}(x, y)}{\partial x_{m}} & =-\frac{B_{m} b}{2 r_{m}}\left(-2 x-e y+2 x_{m}+e y_{m}\right) \\
\frac{\partial I_{m}(x, y)}{\partial y_{m}} & =-\frac{B_{m} b}{2 r_{m}}[-e x-2 y(1+k) \\
& \left.+2 y_{m}(1+k)+e x_{m}\right]
\end{aligned}
$$

\section{References}

Alves, D.R., 2000, ApJ 539, 732

Alonso, A., Arribas, S., Martinez-Roger, C., 1994, A\&A Suppl. 107, 365

Andersen, J., 1991, A\&A Rev. 3, 91

Argyle, R.W., Alzner, A., Horch, E.P., 2002, A\&A 384, 171

Alzner, A., Argyle, R.W., Anton, R., 2009, Commission 26 Circular No 169

Balega, I.I., Balega, Yu.Yu., Maksimov, A.F., Malogolovets, E.V., Pluzhnik, E.A., Shkhagosheva, Z., 2006, Bull. Spec. Astrophys. Obs. 59, 20

Berthier, J., et al. 2002, http://www.bdl.fr/priam/adonis

Bessel, M.S., 2005, ARAA, 43, 293

Bouchet, P., Manfroid, J., Schmider, F.X., 1991, A\&AS 91, 409

Byrd, R.H., Lu, P., Nocedal, J., Zhu, C., 1995, SIAM Journal on Scientific Computing, 16, 5, 1190

Carney, B.W., 1982, AJ 87, 1527

Christy, J.W., Walker, R.L., 1969, PASP 81, 643

Cuypers, J., 1997, ASSL 223, eds. J.A. Docobo et al., 35

Cvetkovic, Z., 2008, AJ, 136, 1746

Cvetkovic, Z., Ninkovic, S., 2008, New Astronomy 13, 587

Delfosse, X., Forveille, T., Ségransan, D., Beuzit, J.-L., Udry, S. et al., 2000, A\&A 364, 217

Devillard, N., 1997, The ESO Messenger, 87, 19

Docobo, J.A., Ling, J.F., 2009, AJ 138, 1159

Docobo, J.A., Andrade, M., 2010, IAU Comm. 26, Inf. Circ 177
Dommanget, J. \& Nijs, O., 2002 Observations et Travaux 54,5 (CCDM)

Edwards, T.W., 1976, AJ 81, 245

ESA, 1997, "The Hipparchos and Tycho Catalogues", ESASP 1200

Fabricius, C., Høg, E., Makarov, V.V., Mason, B.D., Wycoff, G.L., Urban, S.E., 2002, A\&A 384, 180

Halbwachs, J. L., Mayor, M., Udry, S., 2005, A\&A 431, 1129

Hartkopf, W.I., Mason, B.D., McAlister, H.A., 1996a, AJ 111,370

Hartkopf, W.I., et al., 1996b, AJ 111, 936

Hartkopf, W.I., et al, 2000, AJ, 119, 308

Hartkopf, W.I., Mason, B.D., 2010, IAU Comm. 26 Inf. Circ. 170

Hartkopf, W.I., et al., 2012a, AJ, 143, 42

Hartkopf, W.I., Mason, B.D., Worley, C.E., 2012b, "Sixth Catalog of Orbits of Visual Binary Stars", http://ad.usno.navy.mil/wds/orb6.html

Henry, T.J., McCarthy, D.W. Jr., 1993, AJ, 106, 773

Henry, T.,J;, Franz, O.G., Wasserman, L.H., Benedict, G.F., Shelus, P.J., Ianna, P.A., Kirkpatrick, J.D., McCarthy, D.W., 1999, ApJ, 512, 864

Henry, T.J., 2004, in: Hilditch, R.W., Hensberge, H., Pavlovski, K. (eds.) 'The Mass-Luminosity Relation from End to End', ASP Conf. Ser., vol. 318, p. 159

Hutchings, J. B., Griffin, R. F., Ménard, F., 2000, PASP, 112,833

Imanishi, M., Alonso-Herrero, A., 2004, ApJ 614, 122

Kimeswenger, S., Lederle, C., Richichi, A., Percheron, I., Paresce, F., Armsdorfer, B., Bacher, A., et al., 2004, A\&A 413, 1037

Patience, J., Ghez, A.,M., Reid, I.,N., Weinberger, A.,J., Matthews, K., 1998, AJ 115, 1972

Lampens, P., Kovalevsky, J., Froeschlé, M., Ruymaekers, G., 1997, in Proc. JD 14, IAU GA XXIII, Kyoto, 1997

Lampens, P., Strigachev, A., 2001, A\&A 368, 572

Lampens, P., Oblak, E., Duval, D., Chareton, M., 2001, A\&A 374,132

Lampens, P., Strigachev, A., Duval, D., 2007, A\&A 464, 641

Martin, C., Mignard, F., 1998, A\&A 330, 585

Mason, B.D., et al. , 1996, AJ, 112, 276

Mason, B.D., Douglas, G.G., Hartkopf, W.I., 1999, AJ 117, 1023

Mason, B.D., et al., 2002, AJ, 124, 2254

Mason, B.D., Hartkopf, W.I., Wycoff, G.L., Rafferty, T.J., 2006, AJ 131, 2687

Mason, B.D., Wycoff, G.L., Hartkopf, W.I., 2012, "The Washington Double Star Catalogue" http://ad.usno.navy.mil/wds/wds.html

Moffat, A.F.J., 1982, A\&A, 3, 455

Muterspaugh et al., 2010, AJ, 140, 1623

Oblak, E., Cuypers, J., Lampens, P., Seggewiss, W., Chareton, M., Duval, D., 1997, Proc of the ESA symposium "Hipparcos-Venice 97", ESA SP-402, 445.

Persson, S.E., Aaronson, M.A., Frogel, J.A., 1977, AJ 82, 729

Popper, D.M., 1980, ARAA 18, 115

Pourbaix, D., 2000, A\&A Suppl. 145, 215

Roberts, L.C., ten Brummelaar, T.A., 1998, "Poster Proceedings on Fundamental Stellar Properties", IAU 189, 72 . 
Ruymaekers, G., 1999, unpublished Ph. D. Thesis, Catholic Univ. of Leuven

Söderhjelm, S., 1999, A\&A 341, 121

Seymour, D.M., Mason, B.D., Hartkopf, W.I., Wycoff, G.L., 2002, AJ 123, 1023

Ségransan, D., Delfosse, X., Forveille, T., Beuzit, J.-L., Udry, S., Perrier, C., Mayor, M., 2000, A\&A 364, 665

Strigachev, A., Lampens, P., 2004, A\&A 422, 1023

ten Brummelaar, T.A., Mason, B.D., Bagnuolo, W.G., Hartkopf, W.I., McAlister, H.A., Turner, N.H., 1996, AJ 112,1180

Xia, F., Ren, S., Fu, Y., 2008, Ap\&SS 314, 51

Xia, F., Fu, Y., 2010, Chin. A\&A 34, 277 